\title{
Figure S2
}

20

40

60

80

ABE53312.1

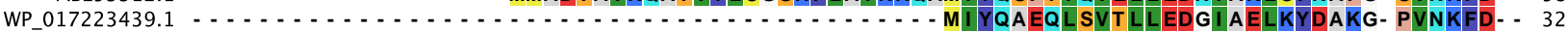

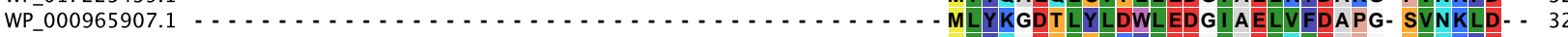

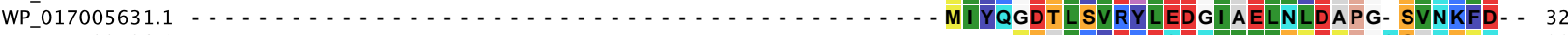

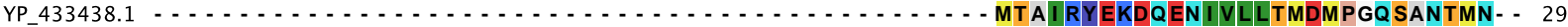

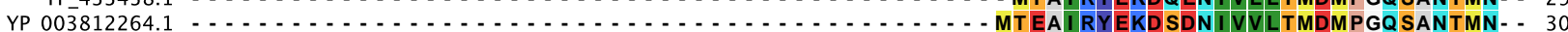

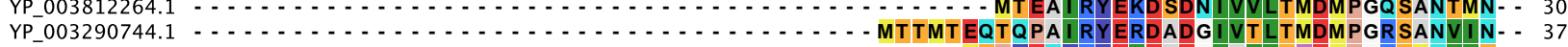

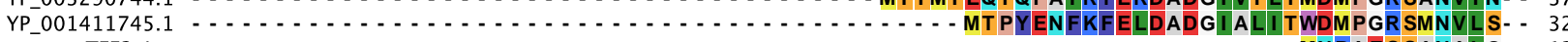

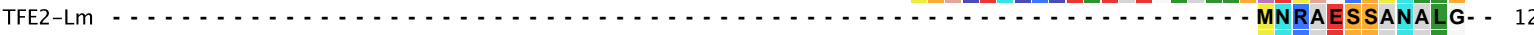

TFE2-Lmex TFE2-Lb TFE2-Lt TFE2-TCCLB WP_019442678.1

NP_718651.1 YP_002416486.1 JW2338

YP_633521.1 EFN74066.1-Ek XP_001988242.1-Ek

P40939-Ek BAB23628.1-Ek
XP_545234.1-Ek NP_996951.1-Ek WP_008448388.1

YP_005996751.1

CBA26305.1 YP_006559517.1

WP_017245866.1

TFE1-Tb

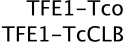

TFE1-Lm

TFE1-Lmex

TFE1-Lt

TFE1-Lb

Conservation

Sequence logo

1
1

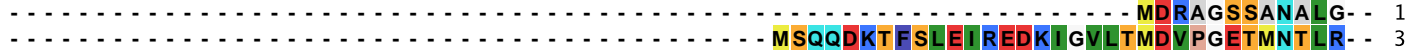

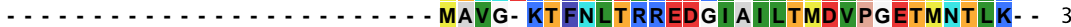
SMT LDVKKEKKA - . . . . . . . . VKKTDSKPVTVSKSDKT VSNDEAVKPATAFSLT IDDQDI AWLAIDVPNEKMNT LQ- . 68 MEMT SAFTLNVRLDNIAVITIDVPGEKMNTLK- - 32 MATKAEELEVKQGFSYQVEG- GVAVITFDLPDSPVNTLS- - 38 MANCRLLGAL- . . . . . . . . VRCSRAN- ANFALKCTGLHQSLRTLTTEANRKYFKCKIVNDVAVITMDSPGVQLNTLN- - 67 MSALRFLSAVGQISKQQLLNNKCSRAHPFSAQLQRRLMSTNLAPAAAASAQKHLHTKVIDGVMVIKIDSPNVKVNSLG- - 78

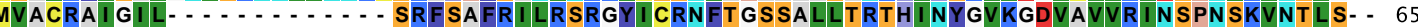

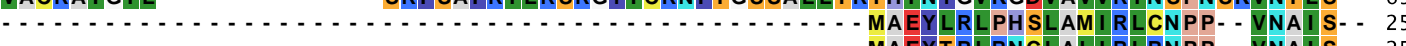

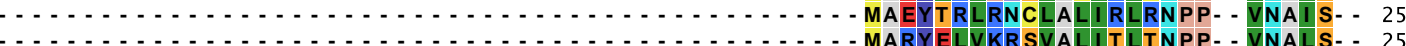
MARYELVKRSVALITLTNPP - VNALS- - 25 MTAEYKVODGVAVITL SNPP. - VNGLG- - 25 .

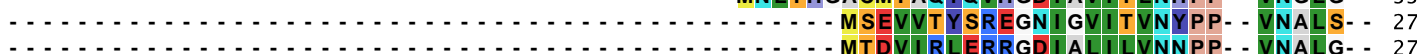

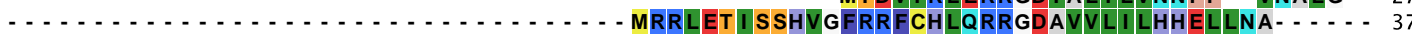
MRRVET I SSHVGFROYCHLERRGAASI VVLHHEPLNA . . . . . . 37

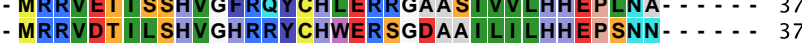
- MKRI IET V SKHLQDN EFVRVERRGVVGL I CMLSSAPPRSHIGGC 43 - MKRLETVSKHLQDNEFVRVERRGGVGLI RMLSSAPPRSHI GGC 43 MQRIEI LSKHLQDNEFVRVERRGGVGLIRMLSSAPPRSHVGGC 43 MRRAETLSKHLQDNAFVRVERRGTVGLI RMLSSAPPRSQVGGC 43 XFXYEERRXG I AVITMDSPG - SVNALG -

o.

ABE53312.1 WP 017223439 WP_000965907. WP_017005631.1

YP_433438.1

YP 003812264.1 YP 003290744 YP_001411745.

TFE2-Lm

TFE2-Lmex

TFE2-Lb

TFE2-Lt

$\begin{array}{r}\text { TFE2 }- \text { TCCLB } \\ - \text { NP } 71942678.1 \\ \hline\end{array}$

NP_718651.1

YP 002416486.1

JW2338

YP 633521.1

EFN74066.1-Ek

XP_001988242.1-Ek

P40939-Ek

BAB23628.1-Ek

XP_545234.1-Ek

NP_996951.1-Ek

WP_008448388.1

YP_005996751.1

CBA26305.1

YP_006559517.1

WP_017245866.

TFE1-Tb

TFE1-TCO

TFE1-TCCLB

TFE1-Lm

TFE1-Lt

TFE1-Lb

Consensus

Conservation

Sequence logo

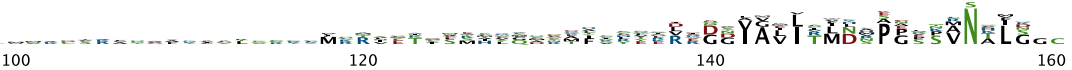
- RETLASLDAALPSIKQN - . ASIQALVLT SAKSTFI VGADITEFLGLFKQDDATLLTWVEQANAV - . . . . . FNKL 124 - TATVASLGEA I GVLEQQ - . - SDLKGLLLRSNKAAFIVGADI TEFLSLFLVPEEQLSQWLHFAN SV - . . . . . . . FNRL 98 - RATLKSLADALSALESE. - . TDVRGLLLT SAKSTFVVGADITEFLDMFSSPDEDLAQWI ADAN SV . . . . . . FSRI 98 - AEFRKAFRDI LQRLQQDK- - DHIAGVI I TSAKKTFFAGGDLRELIQVNKEHAQEFLDMLTTSITQ - . - . . - . PLRQL 97 - AAFTEAFDGVLTRLEKE- - - EKLAGVVVASAKKTFFAGGDLNDLI KVTPENAEQFY- QATNVIIKG- . . . . . HLRRL 96 - EVLAEALAAALDRLEQEK- - DAI SGVI IT SAKSTFLAGADLEGLLALR- DPAEAFR- - RAEQFKA - . - . - . - LLRRL 102 - QSSMADMASIIIEKIMSD-.- DAIKGAVLT SGKDAFCAGADLSMMGGQAGGSGSGSQEDRVKAM YEGNLKFNMLLRGL 106

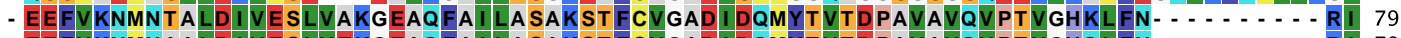

- EEFVKNMNAALDI VESLVEKGEAQFA I LASAKSTFCVGADIDQMYTVTDPAVAVQVPTVGHQLFN - . . . . . . . . RI 79

. EEFVKDMNAALDIVESLVANNEAQFT ILTSAKSTFCVGADIDQMYTI IDPTVAVQVPTLGHMLFN - . . . . . . . . . RI 79

- EEFVTNMNTALDI VESLVAKGDAQFA I LASAKST FCVGADITQMYTVTDPAVAVRVPSIGHTLFN - . . . . . . . . . RI 79 - EAFVGCMQKALDI VEKLVVDKKACVA II A SAKSNFCVGADLDQLYPLTEKKMAAEATRMGHELFD - . . . . . . . . RI 79 - AEFADEI SDLMKEIK- SNSDLQGLVLI S- GKKDSFVAGADVTMIDACETAADAEQLSLEGHRVMG- . . . . . . . E EL 98

. AAFAEEMKA IFEQLKEKQSRVKGLIVHS- LKPDNFI AGADVRMLDACKTADEAQSLARQGQEMFQ- . . . . . . . . AL 134 - AEFA SQVRA II KQLR- ENKELRGVVFVS- AKPDNFI AGADINMI GNCKTAQEAEALARQGQQLMA- . . . . . . . . EI 97

- PET GEAFLRVMMRAE- REPEVKAVVFT S- GKKD SFVAGAKIDFLQTIKTAEEATAISRNGQEGFD- . . . . . . - . KL 103 - KEVI AEV ESLLTEVQ- SNPLVNSAVLIS- GKONNFIAGADI SMLQTFSDAESGYQLVKEAQRVMN - . . . . . . . . IV 132

- AEVSNEFESVIIKDLE- TNAAVKAAVLIS- GKPGCFVAGADI GMLEACKSAEDATLISHGGQIFFD- . . . . . . . RL 143 KELHSEFSEVMNEIW- ASDQIRSAVLIS. SKPGCFI AGADINMLAACKTLQEVTQLSQEAQRIVE. . . . . . . . KL 130 - PTVITEVRNGLQKASLD - - - HTVRA I VI CGANDNFCAGADIHGFKSP. - - TGLTLGSLVDEI QRY - . . . . . . . . . . - 84 - TAVLRGIKDGLQKATTD - - RTVKAI VLCGADGKFSAGADIHSFGEPR- KSDFVLGHIVDEIQRT- . . . . . . . . . . 86 - SAVRHA ISKTMERALSD. - - PKVTAVVIICGENGRFCGGADIREFAGP.- LRGPPLVPLLDAIEAG- . . . . . . . . 85

- LATRTAAVKGI QQALAD - - DAVKAIVI TGAGKAFSGGADIKEFNSPKALTEPSLHTLIATAESS - . . . . . . . . . . 87 - HATRLGIVEGMVRAGDD. - - PGVKAIVI ITGAGKAFSGGADIREFNTPKATQEPTLHAVIQAVESS - . . . . . . . . 87 - LTTRQGI ADGMAQANAD - . AAVKA IVI I TGAGKAFSGGAD ITEFGSPKALQEPNLI SVIKVLESS - . . . . . . . . . 95 HAVRSGLIAALEQGQED - - SEAKVLLLVCEGRTFIAGADIREFGKP. - MQKPGLAELVET YENS - . . . . . . . . . 87 - HAVRKGLLDAFQQADEA - - PEVTAVVLVCEGPTFMAGADI KEFGKP. PQAPSLPEVIIEVI EGC - . . . . . . . . 87

LTVDMRAALLHFFNEADNDS - - SVKCI I IAGEGG- AFSCGIDINDFAASLVDTTKENGVRI PSLPSLT - . . . . . - . TRI 105 LTTNMRAALLHFFNEADADS- - ATRH IVI T GEGD- AFSCGI DVSDFAASVAETNASDDNSVPTLQALC - . . . . . . - NRV 105 LT PKMRAA I QHY FRQAEADP. - AVRRI I LTGEGH - AFSCGVDLADFSASA SQMEY- DGVLI PSLATLT - . . . . . - NMV 104 LCGPMRAQVLRALKT LEADA - DCRFIVLTST SHRFFSSGIDI ANFTECFI SDPT- AQPPVPSLSELT - . . . . . . AAV 112 LCAPMRAQV LRALKTLEADA- - DCRLIVLTST SHRFFSSGIDIANFTECFISDPT-AQPPVP SLSELT - . . . . - AAV 112

CAPMRAQI LRALKALEADA - DCRLIVLTSTSHRFFSSGIDIANFAECFI ADTT-AQPPVPSLSELT - . . . . AAV A 112 CAPMRAQILRALKTLEADA- - DCLFIVLT ST SYRFFSSGIDI ANFTECFVSDLT - AQPPVPSLSELT - . . . . . - AAV 112

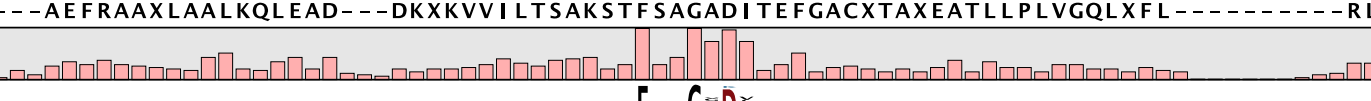

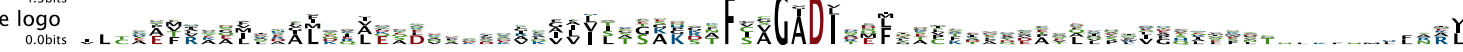


ABE53312.1 EDLPFPTISAINGFALGGGCETIILATDFRVA- - DTNARIGLPETKLGLIPGFGGTVRLPRVIGADNALEWITTGKEQRPD 202 WP_017223439.1 EDLPYPT I SAVNGHALGGGCEA I LATDFRVA- - DTTARI GLPETKLGIMPGFGGTVRLPRLI GADNAVEWITTGKDYKAP 176 WP_000965907.1 EDLPVPT I AAVNGYALGGGCECVLATDYRLA- - TPDLRI GLPETKLGI M PGFGGSVRMPRMLGAD SALEI I AAGKDVGAD 176 WP_017005631.1 EPLPFPT VSVIIAGFALGGGCECI LSTDFRI A- - DTTARI GLPETKLGI I PGFGGTVRMPRLVGADTAMELI TAGKESKAP 176

YP 433438.1 ELLGRPVVAA I NGTALGGGWELALACHAR I ALADER I ELGLPEVT LGLLPGGGGVARLPRYLGLEKALPLLLEGKKLSPQ 177 YP 003812264.1 EKLKAPVVAA INGAALGGGLEI CLACNYR I ALNDRK I KLGLPEVT LGLLPGAGG IVRMT RMLGLEAALPLLLEGKQLAPD 176 YP_003290744.1 ETLGRPVVAAINGTALGGGMELALACHRR IVRDDPSIRLGFPEVTLGLI PGGGGITRLVRLLGLEAAHPYLIEGRQVSPR 182 YP_001411745.1 ETCGKPVVAA INGTALGGGLEVTLACHYR I A SDNPKTQI GLPEAKVGLLPGGGGTQRLPRLIGAQAALPLILQGT SLDPQ 186 TFE2-Lm EQEKFP I VAA INGLALGGGFEMSLACHQRLM - ASTAKVGFPECLLGLLPGGGGTVRTQRLCGLTKTVQWIMTSKQIKPQ 157

TFE2-Lmex EQEKFPIVAA INGLALGGGFEM SLACHQRLM - - ASTAKVGFPECMLGLLPGGGGTVRTQRLCGLTKTVQWIMTSKQIKPQ 157

TFE2-Lb EQEKFPIVAAINGLALGGGFELSLACHQRLM - - ASTAKVGFPECLLGLLPGGGGTVRTQRLCGLTKTVQWIMT SKQIIKAR 157

TFE2-Lt EEEKFPIVAAINGLALGGGFEMSLACHQRLL- - ASTAKVGFPECLIGLLPGGGGTVRTQRLCGLTKTVQWIMT SKQIKPK 157

TFE2-TCCLB EKEPFPVVAA INGLALGGGFELALACHHRLL- - ASDGSVGLPECLLGVLPGAGGTVRLQRLTGLMTAVEWIMT SATAKAQ 157 WP_019442678.1 EALNI PVVAAIHGACLGGGLELALACHIRVCTESTKTALGVPEVMLGLLPGSGGTQRLPRLI GVAKSLDLMLTGKQIRAK 178

NP 718651.1 EALNI PVVAAIHGACLGGGLELALACHQRVCSDDGKTMLGVPEVQLGLLPGGGGTQRLPRLVGITTALDMMLTGKQIRPK 177 YP_002416486.1 SDLPYPVVAAIHGPCLGGGLELALSCDYRVCTDSDKTRLGLPEVQLGLLPGSGGTQRLPRLI GLLPSLDLILTGKQLRAK 214 JW2338 HALPIQV IAAIHGACLGGGLELALACHGRVCTDDPKTVLGLPEVQLGLLPGSGGTQRLPRLIGVSTALEMILTGKQLRAK 177

YP_633521.1 ADFPKPVVAAIHGACLGGGLEWALACDYRI ATDSPKT SLGLPEVQLGLI PGAGGTQRLPALIGVQAALDLILTGKSLKPA 183 EFN74066.1-EK ANSKKPIVAAINGSCLGGGLEVALACHYRLAVNNKQTKLGLPEIMLGLLPGAGGTQRLSQLI SLPT ALDMILTGKTVT AD 212 XP_001988242.1-Ek ERSRKPVVAAISGVCLGGGLELALACHYRI ATKDSKTKLGLPEVMLGLLPGGGGTVRLPKLT SVPTALDMGLTGKQIRAD 223

P40939-EK EKSTKPIVAAINGSCLGGGLEVAI SCQYRIATKDRKTVLGTPEVLLGALPGAGGTQRLPKMVGVPAALDMMLTGRSIRAD 210 BAB23628.1-EK -- QKPVVAA IQGVALGGGLELALGCHYRI A- NAKARVGFPEVMLGI LPGARGTQLLPRVVGVPVALDLIT SGRHISTD 159 XP_545234.1-EK - - EKPVVAA IQGLALGGGLELALGCHYR I A- - HAEAQI GFPEVTLGILPGARGTQLLPRLI GVPAALDLITSGRHVLAD 161 NP_996951.1-Ek - - EKPVVAA IEGVALGGGFELALVCHYRI A- HYKARLGLPEVTLGILPAAGGTQRLPRLIGIPAALELITTGRHVSAQ 160 WP_008448388.1 - - . TKPVVAAIHTVCMGGGLELSLGCHYRVA - MPGAQI ALPEVKLGLLPGAGGTQRLPRVLGLEMALNMIVSGTPVLSD 162 YP_005996751.1 - - GKPVVAAIHSVAMGGGLELAMGCHYRVA- - APGAQI ALPEVKLGILPGAGGTQRLPRAI GLEPALNMIVSGTAVPSE 162

CBA26305.1 - - AKPVVAA I HTVAMGGGLELALGCHYRIA- - APGT SIALPEVKLGLVPGAGGTQRLPRVLGVEPALNMIVSGEPVKSE 170 YP_006559517.1 - - EKPLVAA IHGTALGGGLEMALGCHYRVA- - LNSAKI GLPEVKLGLLPGAGGTQRLPRLTGAQKALEI IT SGEFVGAK 162 WP_017245866.1 - - RKPSVAVI HGTALGGGLEVALGCHYR I A- RSDAKVGLPEVKLGLLPGAGGTQRLPRLAGVEKALEMI VSGQPI GAA 162 TFE1-Tb EQSDKVVIIAAT SGITY SGGLELALAAHYRVAS- - PT SVFCMPEVKLGIVPCGGATQRLPRLIGVRAALDII STGRKVSAK 183

TFE1-TCO EA SNKVVIIAAVGGI AFSGGLELALAAHYRVAL- - PSSSFCMPEVTMGIVPCGGGTQRLPRLI GVRAALDMIATGRKVSAT 183

TFE1-TCCLB ENSVK IVVAA INGLTFSGGLELALAAHYRVAS- - PSSEFSFPEVKLGIVPCGGGTQRLPRLIGVRAAVDMITTSKKLTAR 182 TFE1-Lm AMCPKVCVAVI QGLCSSWGLELALAADYRICE- - MNAHFRFPEVRFGI TPGGGGAQRLVCLVGVPHALNMLCYGRRVYAK 190

TFE1-Lmex AMCSKVCVAI QGLCSSWGLELALAADYRI CE- - VNAQFRFPEVRFGI TPGGGGAQRLVCLVGVPHALNMLCYGRRVYAK 190 TFE1-Lt AMCPKVCVAVIIRGLCSSWGLELALAADYRICE- - LNAQFRFPEVQFGITPGGGGVQRLVCLVGVPHALNMLCYSHRVYAK 190

TFE1-Lb EMCPKVCVAAIQGLCSSWGLELALAADYRI CE- - VNAQFRFPEVRLGITPGGGGVQRLI SQVGVPHALNMLCYGRPVYAR 190

Consensus E-LPKPVVAA I NG LALGGGLELALACHYR I A- -DPKAKLGLPEVKLGLLPGGGGTQR LPRL I GVPAALDM I LTGKQVKAK

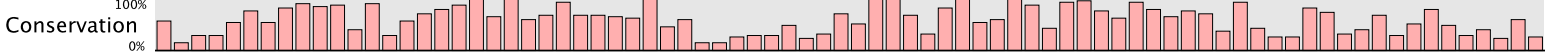

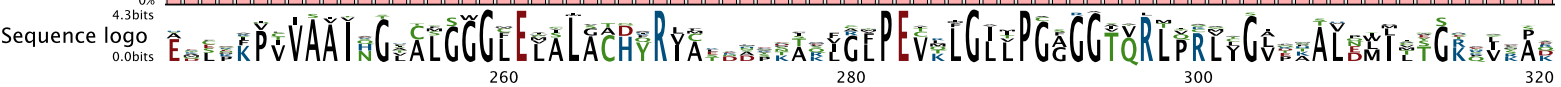

ABE53312.1 DALQV. . . GAIDAVVE. . . . . . . . . . . PENLLAAALQMLKDA . . . . . . . . . . . . L LAEKLDWQARRKR . . . . . . 243

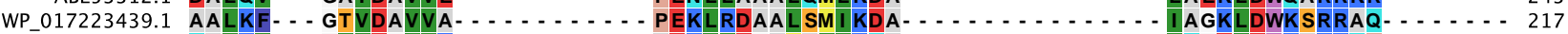

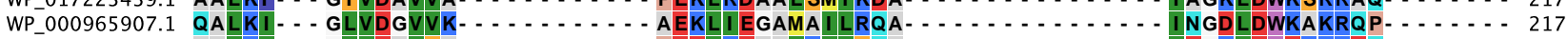

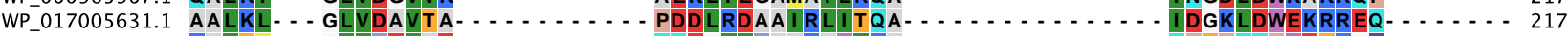

YP_433438.1 KALSM - - GLLHELAE - . . . . . . . . NQEGLIHAARAWIKA . . . . . . . . . . . NPSPQQPWDTKGY . . . . . 218

YP 003812264.1 AAKAA - - GIINEI AD . . . . . . . . . . SPEDMLAKAKAWCKA - . . . . . . . . . . . . NANAQQPWDQKGY . . . . . . 217

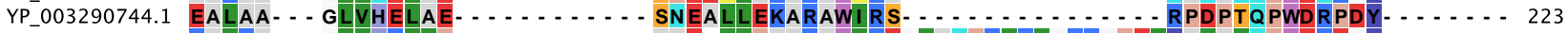

YP_001411745.1 KALKA - . GILHKVVP. - . . . . . . . . . AAE- LI PAAKAWLKEGLAQPKVKLGKKGPEVYAIA A QPWDREGY - . . . . . - 242

TFE2-Lm EAKSA - - GACDV II P. . . . . . . . . . ADDRWNGEHRFYEGVR - . . . KWAGQYLSNKPARLSKRKNI SL . . . . . 208

TFE2-Lmex EAKSA - - GACDAIIS- . . . . . . . . ADDRWNGEHRFYEGVR . . . . KWAGQYLSDKPARLSKRKNISL. . . . . . 208

TFE2-Lb EAKSA - . GACDAVLP. . . . . . . . . . . AEDRWNGEHRFFEGVR . . . . . KWAGQYLSDKPALLSKRKSI SL . . . . . . . 208

TFE2-Lt EAKSS - . GACDVIIIP. . . . . . . . . . A A EDRWT GEHRFYEGVR. . . . . KWAGQHLSDKPARPSKRKSI SL . . . . . . 208

TFE2-TCCLB KAKKA - . GAVNAVIP. . . . . . . . . A A EDRWNGEDRFFNQVR - . . . RWVGG- LVDKPLKPSRGKNLSL. . . . . . 207

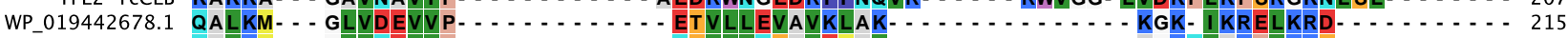

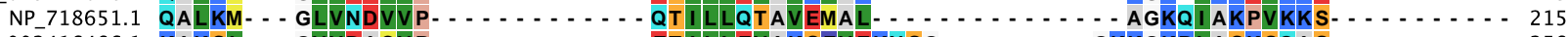

YP 002416486.1 KAKSL. . . GVVDACVP. . . . . . . . . . . . . E ETILLEVAKSFVEKNSG . . . . . . . . GKKGKRLASKSQAS . . . . . . . . . 258

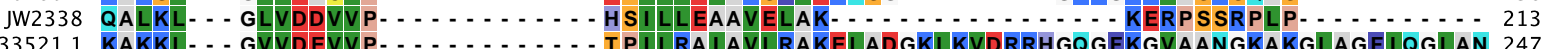

YP_633521.1 KAKKL. - - GVVDEVVP. . . . . . . . . . . TPILRAIIALRAKELADGKLKVDRRHGQGFKGVAANGKAKGLAGFIQGLAN 247

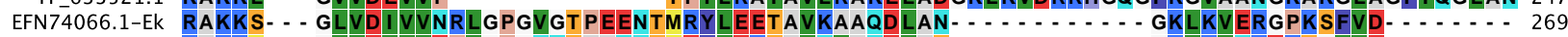

XP_001988242.1-Ek RAKRM - - GIVDLLVDPVGPGQQSAAQN SMDYLERTAIQVANDLAS - . . . . . . . - GKLRVNREKSGLVD - . . . . 280

P40939-EK RAKKM - . - GLVDQLVEPLGPGLKPPEERTIEYLEEVAITFAKGLAD - . . . . . . . . . KKISPKRD- KGLVE- . . . . . . 266

BAB23628.1-EK EALKL- . - GILDVVVVS - . . . . . . . . . DPVEEAIKFAQTVIG - . . . . . . . . . . KPIEPRRIILN - . . . . . 198

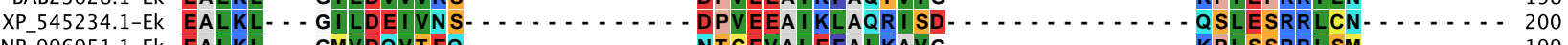

NP_996951.1-Ek EALKL. - - GMVDQVTEQ - . . . . . . . . . . NTCEVALEFALKAVG - . . . . . . . . KPLSSRRLSM . . . . . . 199

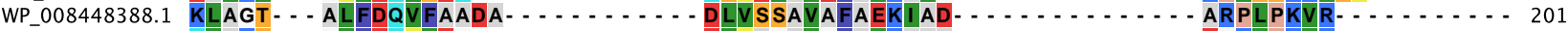

YP_005996751.1 QLAKS . . GLFDQMI EG- . . . . . . . . . . . . DLMAGAI GFALKAAA . . . . . . . . . . . . . EGKLPKLR . . . . . . . . . 199

CBA26305.1 MLAMLPGQKLFPKLSA SPE. . . . . . . . SLAEEALAFARSVAD $\ldots \ldots \ldots \ldots$

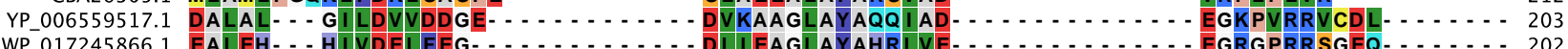

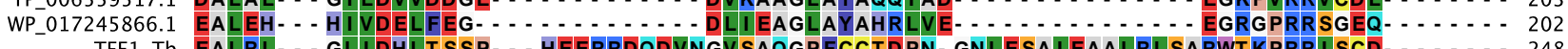

TFE1-Tb EALRL - - - GLIDHLTSSP- - - HEERRDQDVNGVSAQGPFCCTDPN- GNLESAIEAALRLSARWTKPRRI SCD - . . . - . 248

TFE1-TCO EAER I - . - GLIDHLIWR- . . . - NGRSRMN ITNGDPVSSLI AGSEV- SSHNIVLEAALQFAAVWNVPRRI SCS. . . . . . . . 246

TFE1-TCCLB EALRM- - - GLVDHLA SC - . - . - TKKHDKDRND GDST VSNKDGDHLRGNNDHLVAVA I QLST YWKT PRHLSCD - . . . . . - 246

TFE1-Lm EAHQI - - - GLIDTPAY SAPHFWTALREFLEKYVTVAT PGTQDGHGEKATGLSAVEDARAAKAAALHRRRTC- . . . . . - 258

TFE1-Lmex EAYRI - - - GLIDVPAYSAPHFWTALA FFI EQHVTPATPGTPGGHGEKATGLNALEEAQAAKT LALHRRRTC - . . . . . . 258

TFE1-Lt EAHQI - - - GLIDAPAYSAPHFWTALVRFI ENHVTAAVPVTT SEKDKKVTGLNTPEDAQAAKLLALHRRRRR- - . . . - . 258

TFE1-Lb EAYQI - . - GLIDRPPYSAPNLWT SLAEFI EKHVT AAAPI KLGGDGEKT SSLHTLKDAQTAKALALHRRHTC. . . . . . . . 258

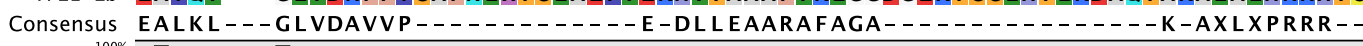

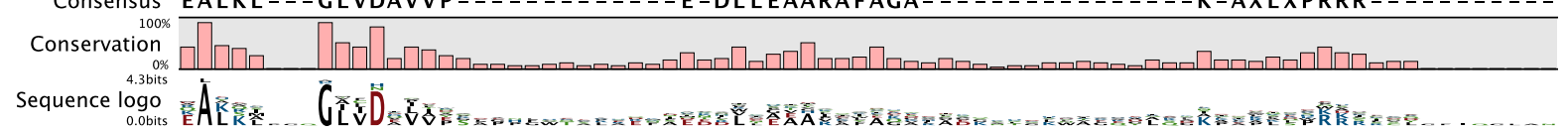




\section{0}

360

380

400

ABE53312.1 - . . KQSPLALPKLEAMMSFTTAKGMVYAVAGKHYPAPMAAVSVVEQAATLDRAGALK- . . . . . . . . . . . VE 299

WP_017223439.1 - . KQAPLGLNKI EATMSFSTALGMVYAKAGKHYPAPTAAVKTIQAAARMDRTGALG - . . . . . . . . . . . AE 273

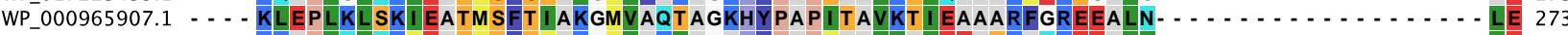

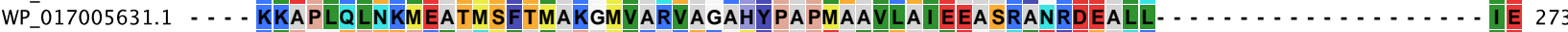

YP $433438.1 \ldots$ KI PGGSPSSPKVAQMLAI APAMLKAKTKGCYPAPEAILSAAVEGAQVDFDTAQK $\ldots \ldots \ldots$

YP_003812264.1 - . - KVPGGTPSTPALAQKLPIIIPAFLLNKTKGCYPAPEAILSAAVEGLQVDFDTAIK - . . . . . . . . . . . . IE 273

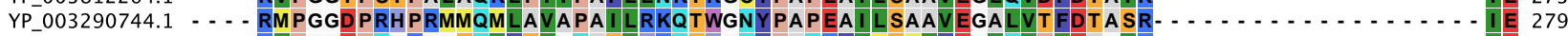

YP_001411745.1 $\ldots$ KVPGGDPNGKGGSQVFT I GNAT LHKQTHGNFPAQKFIMSCVYEGLQVPI IEAGLF $\ldots$

TFE2-Lm - . - MDQVLENT TFGRRKVADET IKMLNKKTRGKYIAQYKALECIMYSATHSNQQGFD $\ldots \ldots \ldots$

TFE2-LmeX - . - MDQVLERT TFGRRKI ADET I KMLNKKT KGKYIIAQYKALECIMYYSATHSNQQGFD . . . . . . . . . . . . . . KE 264

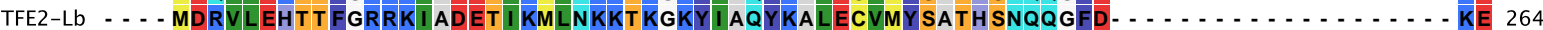

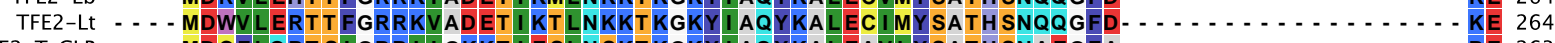

TFE2-TCCLB $\cdots$ MDCFLQRT SI GRR II GKKT I ESLNSKTKGKYIIAQYKALEAVLYSATHSNAEGFA $\ldots \ldots \ldots$

WP_019442678.1 - - L LTGKLLETNKFGRN IMFDQAAKQTFSKTRGNYPAPEAILEVVKLGQEKGFEAGLK - . . . . . . . . . . . . LE 272

NP_718651.1 - - LVNQLLEGTGFGRNIIIFDQAAKQVAKKTQGNYPAPAKIIIDCVRQGIAKGMQKGLE $\cdots \ldots \ldots$

YP_002416486.1 - . AKEKLIISRTGLGRKV IFEQASKKTNQKTRGNYPAADA ILDVIRYGLENGFDKGLQ . . . . . . . . . . . . . . YE 315

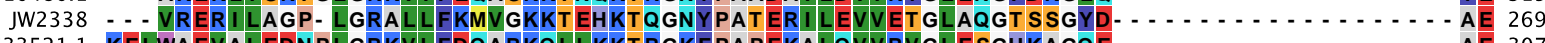

YP_633521.1 KELWAEVALEDNPLGRKVLFDQARKQLLKKTRGKFPAPEKALQVVRVGLESGHKAGQE $\ldots \ldots \ldots \ldots$

EFN74066.1-Ek - - KI I QQVLSFNFVKDQIFGRAKAQVMKAT GGLYPAPLKILEVVRTGLDKGPVVGFE $\ldots \ldots \ldots$

XP 001988242.1-EK … KLQALVMDTDFVKNKIFDTARKQVMKATGGLYPAPLKILEVIRTGVDKGTDAGFE. . . . . . . . . . . . . . . AE 337

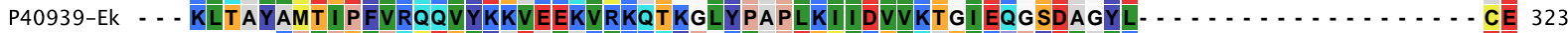

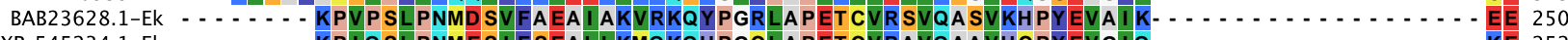

XP_545234.1-EK - . . . . KPIQSLPNMESIFSEALLKMQKQHPGCLAPETCVRAVQAAVHCPYEVGIQ - . . . . . . . . . . . KE 252

NP_996951.1-EK - . . . . - LTTPCPPGLDGIFEAATMQVQKKARGVMAPLACVQAVRAAT - LPYSEGIK - . . . . . . . . . . . RE 250

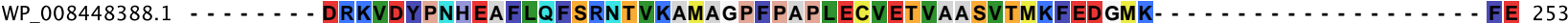

YP_005996751.1 … . . DRKVEHDNPEGFLQFARNTVAAVAKNFPAPGKCVDAVQAAVEKRFDDGIK- . . . . . . . . . . . FE 251

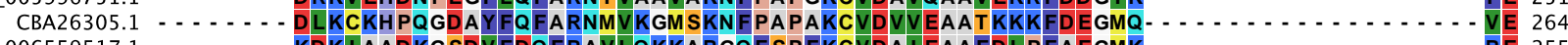

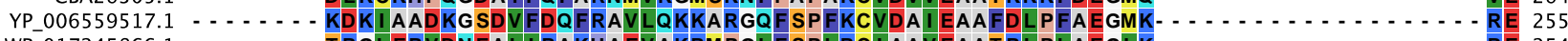

WP_017245866.1 - . . . . TRGLERVDNEALIRAKHAEVAKRMPGLFSPLRCIAAVEAATRLPLAEGLK - . . . . . . . . . . . . . . RE 254

TFE1-Tb - . . . . ATKLGIMLYNSILFRRVGSEI TKKAPKEVSAPLQCLQAIRAATNSASFKEG- . . . . . . . . . . . LAEE 303

TFE1-TCO $\cdots . .$. ARKLGCALTNWAAFCYSERDLARKAYKGSYFQLQCVNAVRAVTKFSSFEEG- . . . . . . . . . . LAEE 301

TFE1-TCCLB $\ldots . .$. NRRLGNSI LNRA LFRWMQREI DKNAPKGVKAPMRCLEA IRASTTVA SF SEG . . . . . . . . . . . . . LQVE 301

TFE1-Lm - .... PAYAQH SLFNRGWY AWMEHKLRD SVPREVRAPYRA IEAVRLAVTHSCRLNGGRSVPSSNGAEQSDAAAAYTVAE 332

TFE1-Lmex - . - . SVYAKHAFLSRGWY AWMEHKLRDAVPREVRAPHRA I EAVKLAVAH SCRLDGSRGVANWGSVDERVAAAAYVAAE 332

TFE1-Lt … - PAY SNVPFFNRGWYAWI EHKLRDSVPREVRAPYCAIEAVKLAVAHSCSPDGRLSVSSLNGSNESNAALAYSAME 332

TFE1-Lb -... - PAYVHLPFFNRGLYAWMKHKLRESVPREVQAPYRAIEAVKLAVAYSDRRGGSGSHPNPNRVNGSVAAAAYAAAE 332

Consensus

Conservation

Sequence $\begin{array}{r}4.36 \text { its } \\ \text { logo } \\ 0.0 \text { bits }\end{array}$ - LEGTXFGRRQI FDQAAKMLXKKTKGNYPAPXKALEAVRAAATHS FDEGLK - -

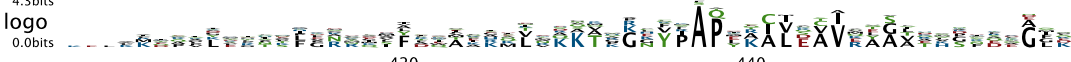
420 440 460

ABE53312.1 NLAFIKLAKTEVATALIGIFLN- - DQFVK . . . . . . . . . . . . . . . . . . . . . . . . GKAKKA - GKL- AKD 338

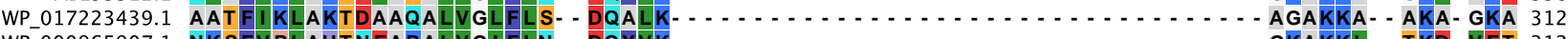

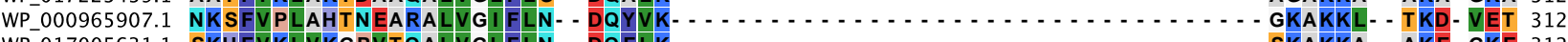

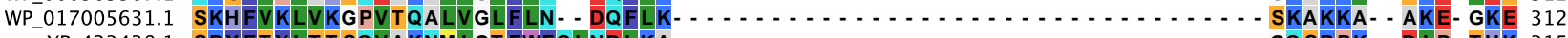

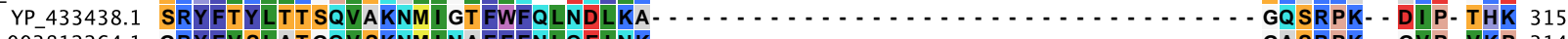

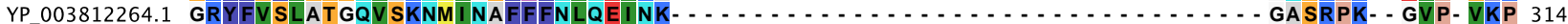

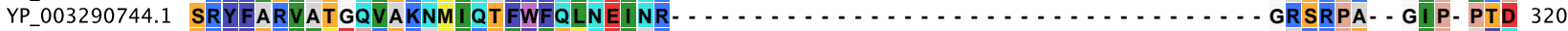

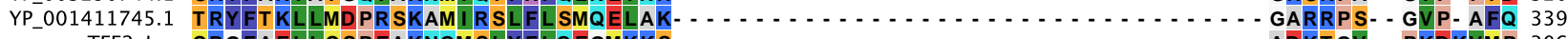
TFE2-Lm CRGFAELLCSPEAKNQMSLYFLQEGMKKS $\ldots \ldots \ldots \ldots \ldots$

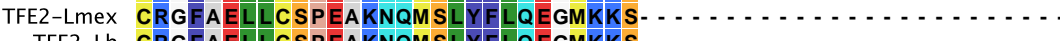

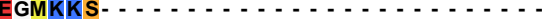

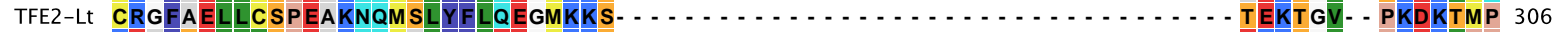

TFE2-TCCLB REAFSELLVTPEAKNQMALYFLDEGMKKS $\cdots \ldots \ldots \ldots$

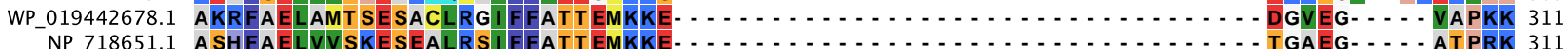

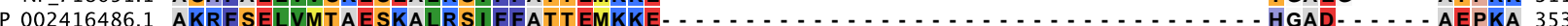

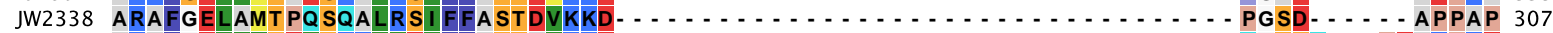

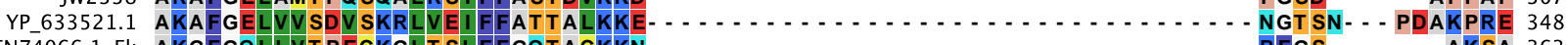

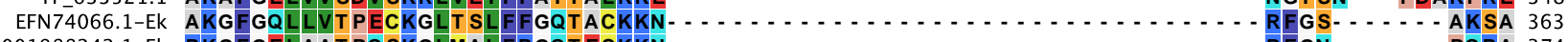

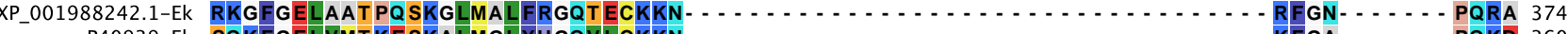

P40939-EK SQKFGELVMTKESKALMGLYHGQVLCKKN $\ldots \ldots \ldots \ldots \ldots$

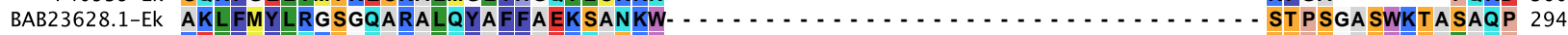

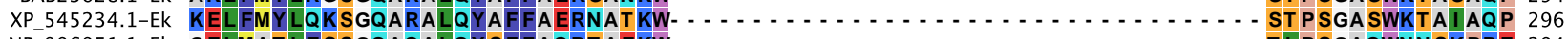

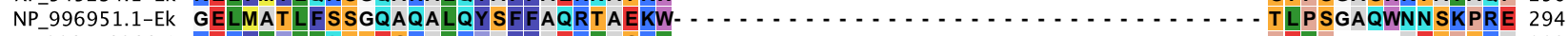

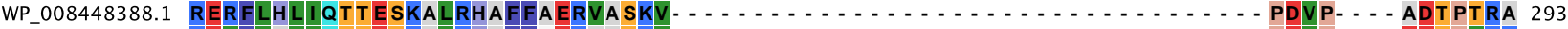

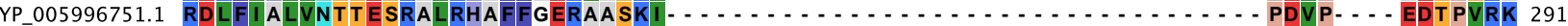

CBA26305.1 REAFINLMFTAESKSLRHLFLAERAASKI $\ldots \ldots \ldots \ldots \ldots$

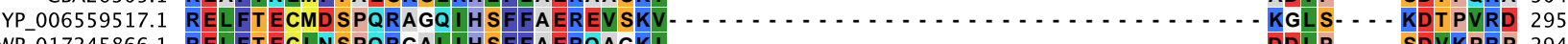

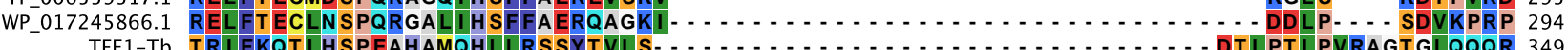

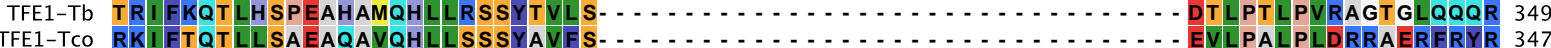

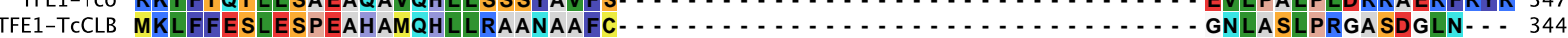

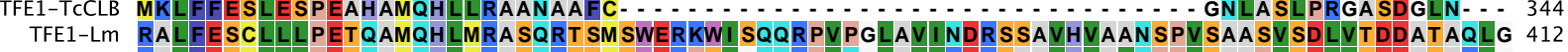

TFE1-LmeX RALFESCLLLPETQAMQHLMRA SQRT SMSWEKPSI GQQRPVQGLAII KDRSAAVHVAVNPPLSAAPV SDVLTDDATARLG 412

TFE1-LT RALFDSCLLLPEAQAMQHLMRASQRT ST SWERKSI GQRHKLPGATRINDHSAVANVMVDPPGSPTAVSDLLSDDATAELG 412

TFE1-Lb RSLFESCLLLPEAQGMQHLLCA SQRT SV SWEKKSLWLSPPVPGLSIMGDVGAAPHFAVKPSA SVA SVYNPSADDATAQVG 412

Consensus RXLFAELLXSPEAKALQHLFFAQXAXKKS - . - . - .

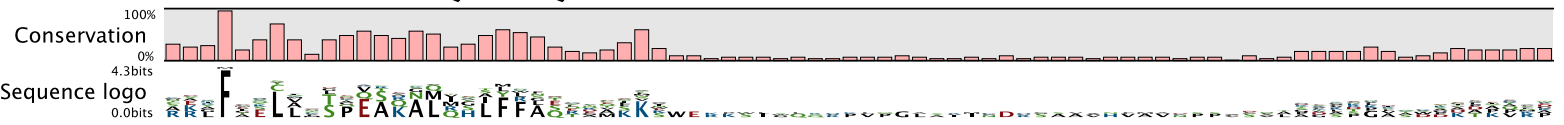


ABE53312.1 VNSAAVLGAGIMGGGIAYQSASKG- TPIVMKDIIAQPALELGLNEAAKILATQVARGRSTPEKMAKVLNNI- - . - TPSLD 412 WP_017223439.1 TNKAAVLGAGIMGGGI AYQSAVKG- TPVIMKDINDAALDLGMATASGLLTTQLKRGRIDGAKLAKVISSI- . - . - TPTLS 386 WP_000965907.1 PKQAAVLGAGIMGGGI AYQSAWKG- VPVVMKDINDKSLTLGMTEAAKLLNKQLERGKIDGLKLAGVISTI- - - - - HPT LD 386 WP_017005631.1 THHAAVLGAGIMGGGI AYQSASRG-VPVLMKDI SQSSLEMGMKEASKLLNKQLERGRINGSKMADVLASI - . . - TPSLH 386

YP 433438.1 MSKVGVLGAGMMGSGI AYACAQRG- LQVVLKDVEQASAEKGKAYSDK I LGKKVSQGRMTESQKQDVLDRI - . . . - TPTAN 389 YP 003812264.1 VKKVGI LGAGMMGAGI AYV SAM SG- I EVVLKDI SDENAQKGKDY SVKLLAKRV SKGQMTQEKADGILAKI- . . - KPTGN 388 YP_003290744.1 TKKVGVLGAGLMGHGI AYVTALAG-MEVVLKDLTLEKAEAGKARIAKLLDERVAKGRLSPEEKQAVLDR I- . - - RTTDR 394 YP_001411745.1 VKKLGI LGAGMMGAGIAYVSAQAG-MEVVLLDTDQANAEKGKAYSEKLLKKALERGKTTQEKADKLLGLI- . . - KPTTN 413 TFE2-Lm VNRVGVI GAGVMGSGI VHYFAKNN- IPVAVKDLTEESVKQGITNVRAEFERAVRRKRMVTAELDGKMALV- - - TGGTT 380

TFE2-Lmex VNRVGVI GAGVMGSGI AHYFAKNN- I PVAVKDLKEESVKRGI TNVRAEFERAVRRKRMVTAELDRKMALV - . . . TGGTT 380

TFE2-Lb VNRVGVI GAGVMGSGI VHYFAKNN - I PVAVKDLKEESVKRGINNVRAEFERAVRRKRMVTAELEKKMDLV. . . . - TGGTT 380

TFE2-Lt VNRVGVI GAGVMGAGI VHYFAKNS- IPVAVKDLTDESVTKGIT SVRSEFEGAVRRHRMATEELEAKMALV- - - - TGGTT 380

TFE2-TCCLB VRTVGVI GAGVMGSGIVHHFANKG- I PVAVKDVKQEAVDKGI EMVRGEFEKAMKRKKITPAIMQERMRIV - - - KGGTS 379

WP_019442678.1 VKKAIVLGGGLMGGGI ANVTATKAKVPVRIIKDIAPQGLLNAQKYTYPLLNKKVKRRFMSKAEMQSQLAMI- - - - TGTTE 386

NP 718651.1 VKKAVI LGGGLMGGGI ASVTTTKAKI PARVKDIINEKGLSNALSYAYKLLDKGVKRRHMTPAARDNLMALM- . - . - TTTTE 386

YP 002416486.1 VKRVGVLGGGLMGAGI SHVSVAKAKVPVRI KDVSNEGVLNALNYNYKLFDKQRKRRILSRAGLESKMLQL- . - . - SGGID 428

JW2338 LNSVGI LGGGLMGGGI AYVTACKAGI PVR I KD INPQGINHALKY SWDQLEGKVRRRHLKASERDKQLALI - . . - SGTTD 382

YP_633521.1 VKKVAVLGGGLMGGGI AYVT SVLQGVPVRVKDKDDAGVGRAMKQVQSI LDERVKRRSLTRREATAKSALV- - - - TAGTD 423

EFN74066.1-Ek AKKIAVIGAGLMGAGIVQVSIDKG-YDVIMKDTNQSGLYRGIGQIQKGLDSAVKRKRISSVEKDKYLSHL- - - - DATLN 437

XP_001988242.1-Ek VETVGVLGAGLMGAGI VQVSVDKG- YKVIMKDAT EPGLARGI GQVQKGLET AVKRKRITALKRDQVM SML- - - . - LPT LN 448

P40939-EK VKHLA ILGAGLMGAGI AQVSVDKG- LKT ILKDATLTALDRGQQQVFKGLNDKVKKKALT SFERDSIFSNL- . . - . TGQLD 434 BAB23628.1-EK VSSVGVLGLGTMGRGIAI SFARVG- IPVVAVESDPKQLDTAKKI ITSTLEKEAS- - - . KSGQASAKPNL- - . - RFSSS 363 XP_545234.1-EK ISSVGVVGLGTMGRGIVVSLAKAK- IPVIAVESDKKQLETADM I ITTLLEKEASK- - MQRSSHPSLGPKP- - - - RLTTS 368 NP_996951.1-Ek IQSAAVI GLGTMGRGI IVVSLARVG- ISVI AVESEKKLLETGRQMVI GMLERDAK- . . - RRGVSASLNLL- . . . - KFSLS 363 WP_008448388.1 IKQAAVI GAGTMGGGI AMNFLNAG- I PVVMLETKQEALDKGLATIIRKNYENTMKKGKLTQEKFDQRMALV- . - . - SGTLA 367 YP005996751.1 VETVAVI GAGTMGGG I AMNFLNAG- I PVTIILETKQEALDRGIIAT I RKN YENSAKKGKLTPEKVEARMALL- . . - KPTLS 365

CBA26305.1 IN SVAVI GAGTMGGG I AMNFLNAG- I PVKMLEMKQEALDRGIAT I RKNYEAQVVKKKLKQDKYEQRM SLL- - - - - STTLS 378 YP_006559517.1 VNSVGIIIGAGTMGGGI AMNFANVG- I PVI IVEMKQEALDKGLGI I RKNYENSAKKGRLTQQQVEDR IAL I - . - . TGSLS 369 WP_017245866.1 IRTAAV IGGGTMGVGIIALSFANAG-VPVKLLEINDEALQRGLQRARET YAASVKRGSLTEDAMEQRLAL I - . . . - AGVTD 368

TFE1-Tb LRKLAVVGCGVVGI GI V IMALR-AGSQVVLLGEDDNECEYALHVI KSELPNDALGYN I SADCVNMYLNNLKVLP- YHGDL 427

TFE1-TCO FNRLIVVGCGPAAVGII IVMALR- RDMCVTLVCENSSECDLALRAIREKLTGDVLKH - - SSTCIEEHLSYLQLVS- FGNDI 423

TFE1-TCCLB I RRFAV I GCGT I GT GVVLLMLR- SQI PVT VVELNEEQCLRARLFMQ EELDREVENGRLLPELCEQRLSMLSLVP- YHADL 422

TFE1-Lm LKKVAVI GAGTMGT SI ALMLLRQSE I EVVLVEADTQRQEVARRSI EDYLRSRVEAHRL SQRRCNDMLHRLRVMGSRIAPF 492

TFE1-LmeX LKKVAVI GAGTMGT SI ALMLLWQS I EV I LVEAEAHRQEVARRT I EDYLRSRVETHRLSRHRCDDMLHRLRVMGSH IVPF 492

TFE1-Lt LKKVAVI GAGTMGT SI ALMLLWQSEI EV I LVEADAQRQEVARHTI EDYLRSRVEAHRLSKHRCDDMLHRLLLRGSVIEPF 492

TFE1-Lb LKMVAVI GAGTMGT SI ALMLLWKSEIEVI LVEMDVQRQEVARHT IEDYLRSRVEAHRLSAHRRDDMLRRLRVMGSLITPF 492

Consensus VKKVAV I GAGTMGGG I AYVFAXKG - I PVXLKD I XQEALERGXAX I RKLLEKAVKRGRLTXXXLDDXLALL- - - - TGTXS

Conservation

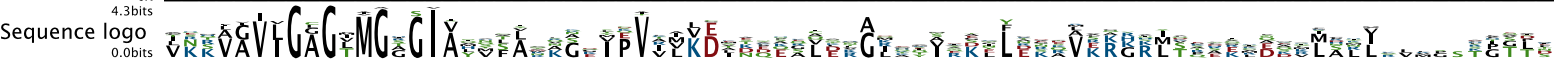

ABE53312.1 YAAIKHSDIVVEAVVEHPKVKATVLAEVED- - YVAEDAIIASNTSTISINLLAKSLKKPERFCGMHFFNPVHKMPLVEIII 490 WP_017223439.1 YNSVDEAD I I VEAVVENPK I KAAVLAELED- - NVSADTV I T SNT ST I P I NVLAKNLKRPEQFCGMHFFN PVHKMPLVEI I 464 WP_000965907.1 YAGFPRVDIVVEAVV VENPKVKKAVLAETEQ- - KVRQDT VLASNT ST I PI SELANALERPENFCGMHFFN PVHRMPLVEI I 464 WP_017005631.1 YAGI EQADV I VEAVVENPT I KAAVLSEVES- - LI SEDAVLT SNT ST I PI DLLAKSLKRPENFCGMHFFN PVHHMPLVEVI 464

YP_433438.1 ISLMEGCDLI I EAV FEN SELKAKVTQESEP- - MLRPEGVFASNT ST I PI TQLAQASSDPAKFIGLHFFSPVDKMQLVEI I 467 YP 003812264.1 AADLAGCDLVVEAVFEKRELKASVTKEAEA - - VMDANGTFASNTSSLPIT GLAEASVRPEKFI GLHFFSPVDKMPLVEII 466 YP 003290744.1 VEDLAGCDLVIIEAVFENRDVKAQVIIRETEA- - VLPEDAVFGSNTSTLPITSLAAYSRRPDRFVGIHFFSPVHRMRLVELI 472 YP_001411745.1 YDDLKGADLVIEAVFESRDIKAEVTKKAEP. - MLAAGGIYGSNT STLPI T GLAEASAKPDNFI GI HFF SPVDKMQLVEII 491 TFE2-Lm NEVFRDADVI VEAAVEVMDIKKKVIQQLEKDGI LHSKSLFATNT SSLSLTEMQTVAKCPHNIVGMHFFNPV SKMPLVEVI 460

TFE2-Lmex NEVLRDADV I VEAAVEVMDI KKKV IQQLEQDGVLHSKSLFATNTSSLSLTEMQTMAKCPHN IVGMHFFNPVSKMPLVEVI 460

TFE2-Lb NEVFRGVDVVVEAAVEVMDI KKKVI QQLENEGI LN SKNLFATNTSSLSLTEMQTVAKCPQNIVGMHFFNPV SKMPLVEVI 460

TFE2-Lt NEVFRGADV IVEAAVEVMDTKKKVI QQLENEGILT SKNLFATNT SSLSLTEMQT VSKYPHN IVGMHFFNPV SKMPLIEVI 460

TFE2-TCCLB SSI FQGMDVIVEAAV EVMDI KKN I IRDLEKEGIINGKN LFATNT SSLSLTEMQTV SKY PEN I VGMHFFN PV SKMPLVEVI 459 WP_019442678.1 YT GVKSADIVIIEAVFEDLKLKHQMVADIEA- - NCNENT I FA SNT SSLPI TQI ASEAKRPENVI GLHYFSPVDKMPLAEI I 464

NP_718651.1 YKGVKDADIVVEAVFEDLALKHQMVKDIIER- - ECGEHTI FASNT SSLPI GQI AQAASRPENVI GLHYFSPVEKMPLVEVI 464

YP 002416486.1 FT SFNHTDVVIEAVFEDLDLKQSMVADIEA- - NAKPSTI FATNTSSLPIHKI AEKAQRPENVVGLHYFSPAEKMPLVEVI 506

JW2338 YRGFAHRDLI I EAVFENLELKQQMVAEVEQ- - NCAAHTI FASNT SSLPI GDI AAHATRPEQVIGLHFFSPVEKMPLVEII 460

YP_633521.1 YSGFKSADLVIEAVFEDLKLKHRI I AEVEA- - VTGDQT I FASNT SSIPITELAKGSRRPAQVIGMHYFSPVHKMPLLEII 501

EFN74066.1-EK YNSFKDVDVVI AVFEDIGIKHKVLKEIES- - IVPQHCVIATNTSAIPITKIAAGSSRPDKVI GMHYFSPVDKMQLLEIII 515 XP_001988242.1-EK YKDFEKADIVIEAVFEDIQI KHRVIIKELEA- - VVPEHCI I ASNTSAIIPI TQIIAAGSSRPDKVVGMHYFSPVDKMQLLEII 526

P40939-EK YQGFEKADMVI EAVFEDLSLKHRVLKEVEA - - VI PDHCIFASNT SALPISEIAAVSKRPEKVI GMHYFSPVDKMQLLEII 512 BAB23628.1-EK TKELSSVDLVIEAVFEDMNLKKKVFAELSA- - LCKPGAFLCTNT SALDVDDI ASSTDRPQLVIGTHFFSPAH IMRLLEVI 441 XP_545234.1-EK MKELGGVDLVIEAVFEEINLKKRVFAELSA- - ICKPEAFLCTNT SALDIDEI ASSTDRPHLVI GTHFFSPAHVMKLLEII 446 NP_996951.1-EK LQDLKDVDLVIEAVFEDMALKKQ IFRELSR- - VCRPATLLCSNT SGLDVDALADVTDRPQLVAGMHFFSPAHVMKLLEVV 441 WP_008448388.1 YEDI GQAD IVVEAV FEDMGVKETVFRKLDE- - VMKPGA I LASNT STLDLNQI ANFTKRPQDVI GTHFFSPANVMKLLEIV 445

YP_005996751.1 YDDIRDADLVVEAVFEDMGVKET VFKTLDA- - VAKPGAILASNT STLDVDRI AGFTRRPQDVVGMHFFSPANVMKLLEVV 443

CBA26305.1 YDDLTGTDMV I EAVFEELGVKEAVFKELDR- - VMKPGAI LASNT ST LDVNKI ANFTQRPEDVVGLHFFSPANVMKLLEVV 456 YP_006559517.1 YDDFKSVDLVI EAVFENMAVKKEI FAKLDA- - ICKPGT I LASNT ST LDVDEI ASATRRPEDVVGMHFFSPANVMKLLENV 447 WP_017245866.1 YGALADADVVVEAVFEEMGVKQQVFEQLDA- - VCKPGAILASNT SSLDLNAIAAFTRRPEDVVGLHFFSPANVMRLLEVV 446

TFE1-Tb QTVLQDVDVMAECI VGDLDTKRQV FTMLTD - - LCPPHCVLATCCSSLELREFVKVSRRPEKVVGMYFAPPVHNVPFLEVT 505

TFE1-TCO GAALRDGDIVMDCTVGDLRQKREI FIQLEA- - MCPPHCVLATCCSTVSVGELAACVQRPERVVGMFFAQPVDDVPFLEVV 501

TFE1-TCCLB VQALKEVDMVLECVGENPALKKQVFTRLSE- - VCPPHCILAT SASTT SI GELAKVTRRPEKVI GLHFIPPSDVTPLLEIV 500

TFE1-Lm PPVLADADLVFECAPEVAAIKQN I LAFLDS- - VCKRSTILATGSSAQDVNELAAVTQRPGQVLGI HFFPPANESPLVEVI 570

TFE1-LmeX PPVLADADLVFECAP EVAAIKQN ILAGLDS- - VCKRSTIFATGSSAQDVNELAAVTQRPGQVLGIHFFPPANESPLVEVI 570

TFE1-LT PSALADADLIFECAPEVESI KQNIFAGLDA - VCKRSTI ILATSSSAQDVNELAAVTQRPGQVLGVHFFPPANEAPLVEVI 570

TFE1-Lb PPVLADADLVFECAPEVAAIKQSIFTRLDS- - VCKRSTILATSSLALEVNELAAITRRPGQVLGMHFFPPANESLLVEVI 570

Consensus YEDLKDADLV I EAVFEDMD I KKQVFAELEA- - VCKPHT I LASNTSXLP I TELAAVTKR PENVVGMHFF SPVHKMP LVEV I

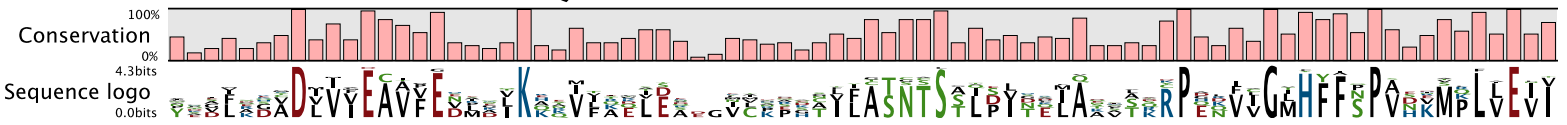


ABE53312.1 RGEHSSEET IASVVAYASKMGKT PIVVNDCPGFFVNRVLFPYFAGFNGLLADGADFAAVDKVMEKQFGWPMGPAYLLDVV 570 WP_017223439.1 RGEKSSDET I AT TVAYAAKMGKT PI VVNDCPGFFVNRVLFPYFFGFSKLI ADGADFVAVDKVMEKQFGWPMGPAYLLDVV 544 WP_000965907.1 RGEKSSDET I AKVVAWASKMGKT PI VVNDCPGFFVNRVLFPYFAGFSQLLRDGADFRK I DKVMEKQFGWPMGPAYLLDVV 544 WP_017005631.1 RGKDT SDETVSRVVAYAAKMGKSPVVVNDCPGFFVNRVLFPYFAGFSLLLRDGADFTKIDKVMEKKFGWPMGPAYLLDVV 544

YP_433438.1 VGEST SQESLAKAFDFVMQI GKVPI VVNDSRGFFT SRVFGTFVNEGVAMLAEGIHPAAIENAAQLAG-MPVGPLALSDEV 546 YP_003812264.1 CGKKT SQET LAHALDYVLQ I KKTPI VVNDARGFFTTRVIIGT YVNEGLRLLAEGAAASSI EMAAARAG- FPIGTLA I SDEL 545 YP_003290744.1 RGRQT SDEALAKAFDYVRKI GKTPI VVNDSRGFYT SRVFGT Y LNEGLALLAEGQHPRAI EVAGRQAG- MPI GPLAVADEV 551 YP_001411745.1 MGKKT SDETLAKAMDYVKQIRKTPIVVNDSRGFYT SRCFGT YVGEGLAMLGEGVPPAMI ENVGKMTG-MPMAPLALNDEV 570 TFE2-Lm KGKST STEAAAA IFNLALKTGKI PI I VNDGPGFLVNRI LGVYMAEAGRLAVDERCHPACVDEAI LAFGMPMGPFRLLDEV 540

TFE2-Lmex KGKST SSEAAAAIFNLALKT GKI PI I VNDGPGFLVNRI LGVYMAEAGRLAVDERCHPACVDEA I LAFGMPMGPFRLLDEV 540 TFE2-Lb KGKST SAEAAAA I FNLALRT GKMPII VNDGPGFLVNR ILGVYMAEAGRLAVDERCHPSCVDEA ILAFGMPMGPFRLLDEV 540

TFE2-Lt KGKKT SAEAAAA IFNLALKT GK IPI I VNDGPGFLVNRI LGVYMAEAGRMVEDERCHPACVDEA ILAFGMPMGPFRLLDEV 540

TFE2-TCCLB KGKKT SKEAAA I IFN ALRT GKKPII VQDAPG FVVNRI LGVYMAEAGRLAVDDKVDLARIDKA ILDFGM PMGPFRLLDEV 539 WP_019442678.1 THEGT SDQT I STTVEFARKQGKTPIVVKDGAGFYVNRI LAPYMNEAARLVLEQEPIEAVDKALVDFG- FPVGPVTLLDEV 543

NP 718651.1 AHAKT SPETIATTVAFARKQGKTPIVVQDGAGFYVNRILALYMNEAAQLLLEGQSVEHLDKALVKFG- FPVGPITLLDEV 543 YP 002416486.1 PHETTSEET I STVVALAKKQGKTPIVVKDTAGFYVNRI LAPYMNEAAHLLLANEPIEKLDSTLLDFG- FPVGPITLLDEV 585 JW2338 PHAGT SAQTIATTVKLAKKQGKTPIVVRDKAGFYVNRI LAPYINEAIRMLTQGERVEHIDAALVKFG- FPVGPIQLLDEV 539

YP_633521.1 THAGTADWVTATCVEVGRKQGKTVIVVNDGPGFYTSRILAPYMNEAAYLLAEGADI AELDRALVEFG- FPVGPITLLDEV 580

EFN74066.1-EK THKGTSEET I RTAVDVGLKQGKIVIITVGDGPGFYTTRILSAMLSEA IRLMQEGVDPVQLDKLTKAFG- FPVGAATLSDEV 594 XP_001988242.1-Ek THPGT SKDTVAQAVAVGLKQGKVVIITVGDGPGFYTTRI LATMLSEAIRLLQEGVDPKELDQLTKKFG- FPVGAATLADEV 605

P40939-EK TTEKT SKDT SA SAVAVGLKQGKV I I VVKDGPGFYTTRCLAPMMSEVIRILQEGVDPKKLDSLTTSFG- FPVGAATLVDEV 591 BAB23628.1-EK PSRYSSPTTI ATVMSLSKRI GKI GVVVGNCYGFVGNRMLAPYYNQGYFLI EEGSKPEGVDGVLEEFG- FRMGPFRVSDLA 520 XP_545234.1-EK PSQYSSPTT I AT VMNLSKK I KK I GVVVGNCFGFVGNRMLKPYYNQT YFLLEEGSRPEEIDQVLEEFG- FKMGPFRVSDLA 525 NP_996951.1-Ek CGPRSSKEAIATAMSLGKRMGKV SVAVGNCPGFVGNRMLMPYLEQATFLLEEGATPQQIDKALEDFG- FAMGVFRMSDLA 520 WP 008448388.1 RGAQT GKDVLATALALSKKLKKT GVVSGVCDGFI GNRMI EQYSRQAGFLLEEGALPEQVDKAVEKFG- FAMGPFRMGDLA 524 YP_005996751.1 RGKATAKDVLAT VMKLAKAI KKT AVVSGVCDGFI GNRMI EQY SRQAGYLLDEGALPEQVDRAVEKFG- FAMGPFRMGDLA 522

CBA26305.1 RGEKTAKDVMAT VMSVAKKI KKTAVVSGVCDGFI GNRMI IEQYGQGGFLLDEGCTPEQVDKAMERFG- MAMGPFRMGDLA 535 YP_006559517.1 RGSKT SDEVKATVMAVAKK I KKVGVLVGNCHGFVGNRMLHRRGAEAMT LVN EGATPQQVDKVLTDLG- YPMGQFAMSDLA 526 WP_017245866.1 RGERT SDEVLAAAMA I GKQLKKV SVVVVGVCDGFVGNRMVFQYGREAEFLLEEGATPQQVDAALRNFG-MAMGPFAMRDLS 525 TFE1-Tb RGYRTDHTTLQRAIHVGRLFHKATI LTRDVGFSIIISRIFFA I LYQALSMLEQGAFPVDIDRAMRH- FGFRLGIFAMEDLA 584 TFE1-TCO QAEHTGQRALQCA I QAGRLFYKATIILSRDASVSVGVRIFSAFLYQALSMLEEGVFPIEIDQVMQR- FGFRLGIFALDDLV 580

TFE1-TCCLB QGSCTDQFT I LQALRVGHLFHKAV I LTRDLGACLTTRLFSAGLYQAFAMLEEGAFPVEVDRALRR-FGFRLGVFALEDLA 579 TFE1-Lm RGAAT ERWVVELVMRLLCQLDKYPI LSVSRHGYVGTRLLLTALYQAYAMLEDGCFPLQIDNVLRKNFHFTRGLFELEDIM 650

TFE1-LmeX RGAATERWVV ERVMHLLCQLDKYPILSVSRHGYVGTRLLLTALYQAYAMLEDGCFPRQIDSVLRKDFHFTRGLFELEDIM 650 TFE1-Lt RGSATQRWVVERMMQLLCQLDKYPILSVSSQGYVGTRLLLTGLYQAYAMLEDGCLPLQIDSALRKNFHFTRGLFELEDMM 650

TFE1-Lb RGTATEQWVVERIMHLLCQLDKYPILSVSRHGSVGTRLLLTALYQAYAMLEDGCFPLQIDRALRKNFHFTRGLFELEDII 650

Consensus RGXKT SDET I ATAXALAKK I GKTP I VVNDCPGFVXNR I LGPYLNEAGRLLEEGADPEQI DKALEKFG - FPMGPFRLLDEV

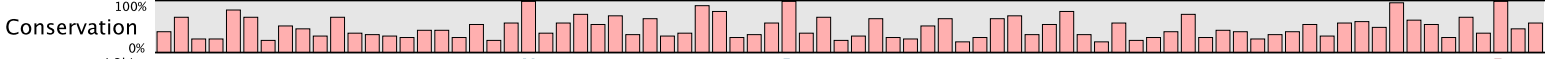

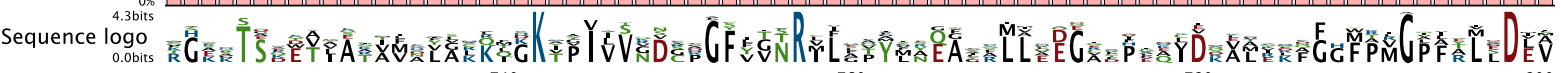

ABE53312.1 GIDTGHHAQAVMAEG- . FPDRMGKNGT - . . . . . . . . . . . . . . . . DAIDIMFENK- RLGQKNSKGFYAYSVD 621 WP 017223439.1 GIDTGHHAAAVMAEG- WP_017223439.1 GIDTGHHAAA MAEG- FPERMAKTGK - $\ldots$

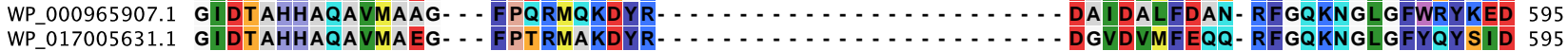

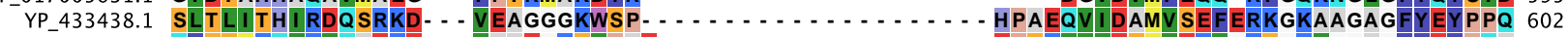

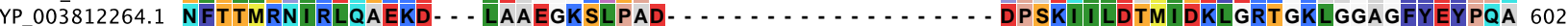

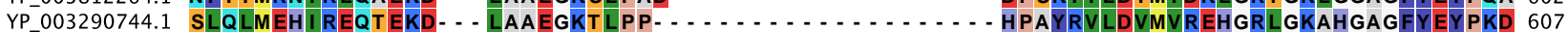

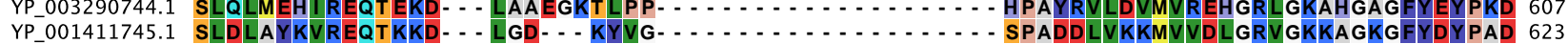

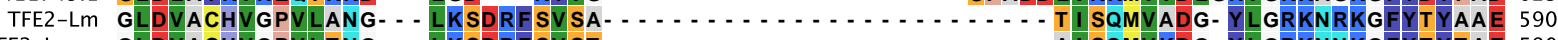

TFE2-Lmex GLDVACHVGPVLTNG- - LKSDRFSVST . . . . . . . . . . . . . . . . . AI SQMVKDG- YLGRKNNKGFYTYTAE 590

TFE2-Lb GLDVACHVGPVLTNG. - LKSDRFGVSP. . . . . . . . . . . . . . . . . . . . . . SI SLMVKDG- HLGRKNNKGFYNYTAD 590

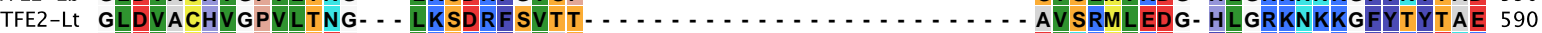

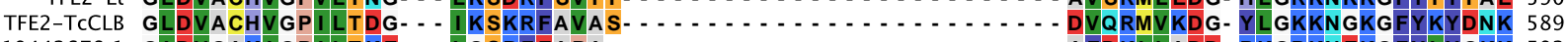

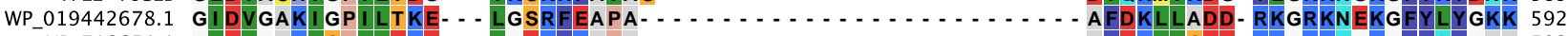

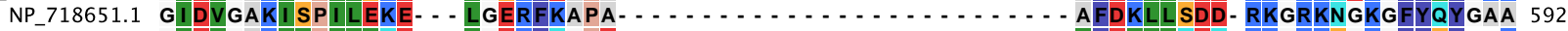

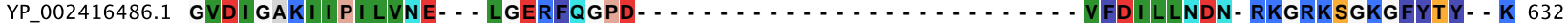

JW2338 GIDTGTKIIIPVLEAA - . YGERFSAPAN - . . . . . . . . . . . . . . . . V V SSILNDD- RKGRKNGRGFYLYGQK 589

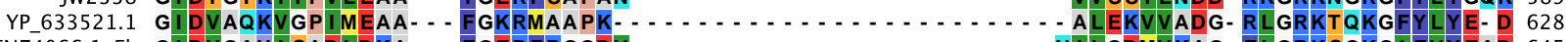

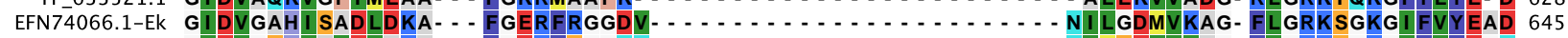

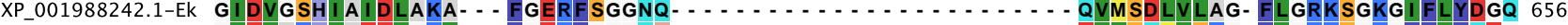

P40939-EK GVDVAKHVAEDLGKV. . FGERFGGGNP. . . . . . . . . . . . . . . . . . . . . ELLTQMVSKG- FLGRKSGKGFYIYQEG 642

BAB23628.1-EK GLDVGWKVRKGQGLTGPSLPPGTPTRKRG - . . . . . . . . . . . . . . . . NTRYSPI ADMLCEAGRFGQKTGKGWYQYDKP 580

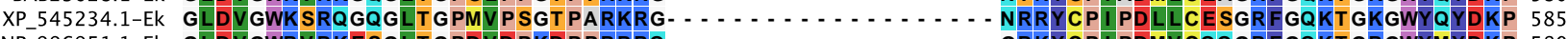

NP_996951.1-EK GLDVGWRVRKESGLTGPDVDPKDPPRRRQ - . . . . . . . . . . . . . . . . . . GRKYCPI PDMVCQQGRFGQKT GRGWYMYDKP 580

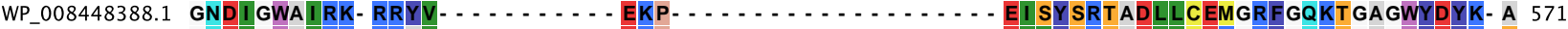

YP 005996751.1 GNDI GWAIRK- RRAV . . . . . . . . . . . DKP . . . . . . . . . . . . . . . . . . . DIVY SKTADLLCEKGRYGQKTGAGWYDYK- T 569

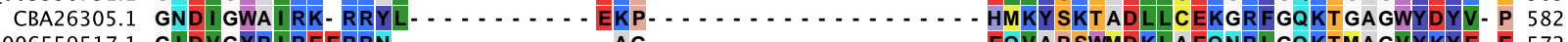

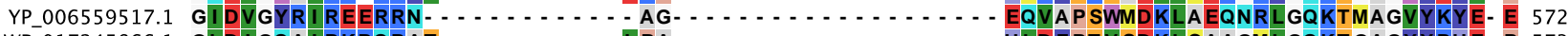

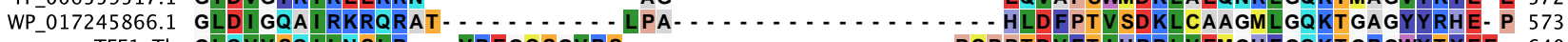

TFE1-Tb GLQVVSQILNSLR . . VREGCSGVRS - . . . . . . . . . . . . . . PCPPTDVFT I HRRLVEMGHFGQKTGRGWYT YEE- 640

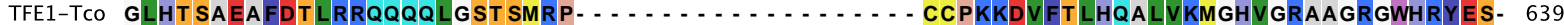

TFE1-TCCLB GLDFTAEIRTRLQ - . . . . KNNTT - . . . . . . . . . . . . FSSQADVYT IPRLLVEEGRLGQKRGLGWYNYEK- 630

TFE1-Lm GLDVMAMARA SMMAVAARA SGSSVPPPKRVGIEPSVPSAFELSNAWCLPSRPVFDIPNALVAAGALGRKTREGWYKYLTP 730

TFE1-LmeX GLDVMAMART SMLEVAARANGSSVPPPKRVGVEPSVPSPFELSNAWFLPSRPVFDI SNALIATGTLGRKTREGWYKYLTP 730

TFE1-Lt GLDVMAMARA SMLAAAARAKGSAAAPPKRSGLGPSI PSAFELSNAWCLPSRHVFDI PNALVAAGALGRKTREGWYKYLTP 730

TFE1-Lb GLDVMGMARA SMLAAVARA SATPPPLPKRVGIIEPSAPSAVEI SNAWWLPSRPVFDI PDALIIAAGALGRKTRTGWYNYVTP 730

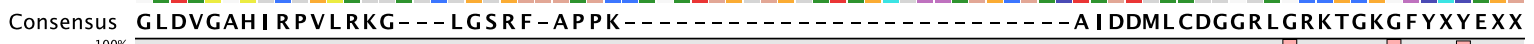

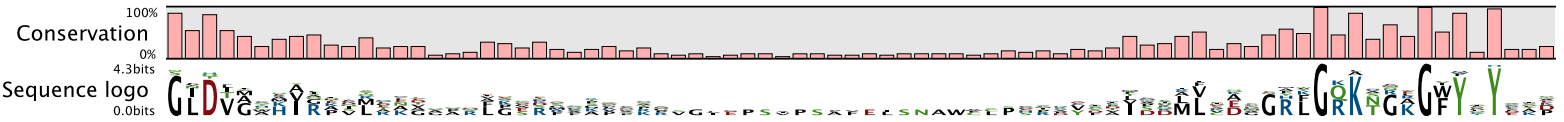




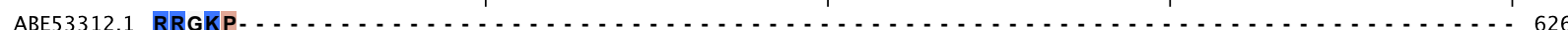

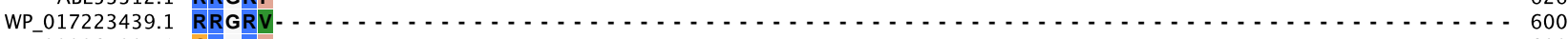

WP_000965907.1 SKGKP. . . . . . . . . . . . . . . . . . . . . . . . . . . . . . . . . . . . . . . . . 600

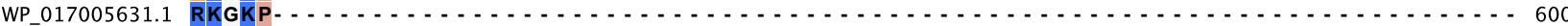

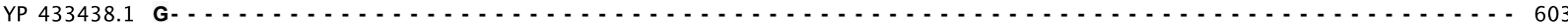

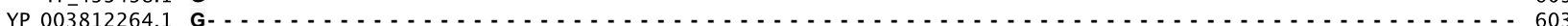

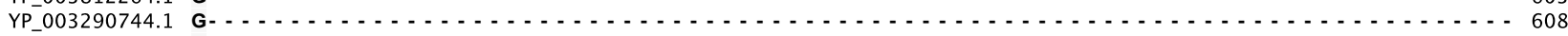

YP_001411745.1 G. . . . . . . . . . . . . . . . . . . . . . . . . . . . . . . . . . . . . . . . . . . . . . 624

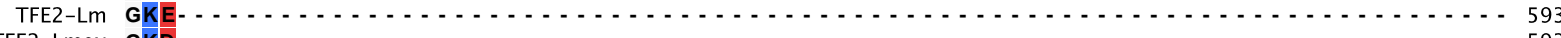

TFE2-Lmex GKD . . . . . . . . . . . . . . . . . . . . . . . . . . . . . . . . . . . . . . . . 593

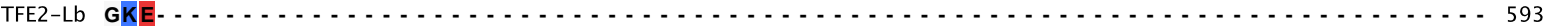

TFE2-Lt GKE. . . . . . . . . . . . . . . . . . . . . . . . . . . . . . . . . . . . . . 593

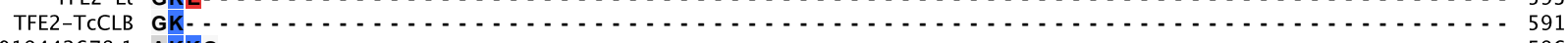

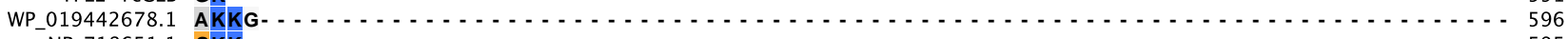

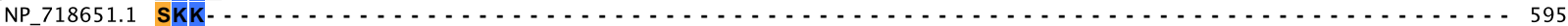

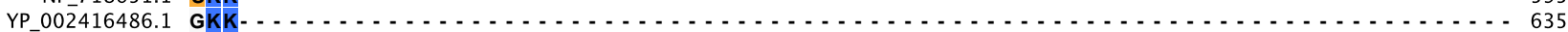

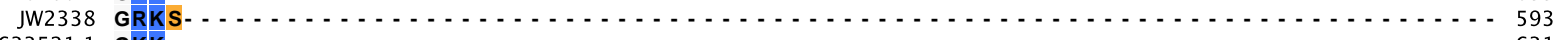

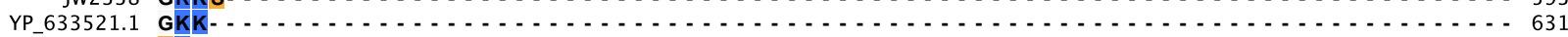

EFN74066.1-Ek SK-. . . . . . . . . . . . . . . . . . . . . . . . . . . . . . . . . . . . . . . . . . . . . . . . . .

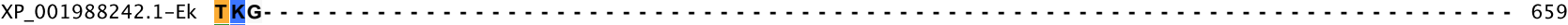

P40939-Ek VK . . . . . . . . . . . . . . . . . . . . . . . . . . . . . . . . . . . . . . . . . . . . . . .

BAB23628.1-Ek LGRIH. . . . . . . . . . . . . . . . . . . . . . . . . . . . . . . . . . . . . . . . . . . 585

XP_545234.1-Ek LGRIH. . . . . . . . . . . . . . . . . . . . . . . . . . . . . . . . . . . . . . . . 590

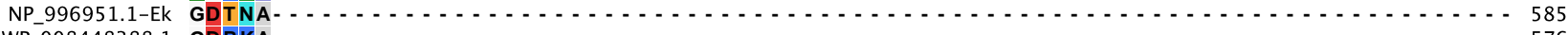

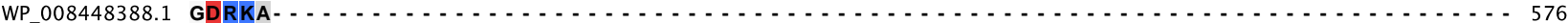

YP_005996751.1 GDRKP. . . . . . . . . . . . . . . . . . . . . . . . . . . . . . . . . . . . . . . . . . 574

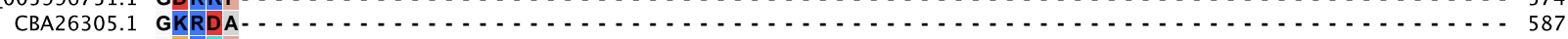

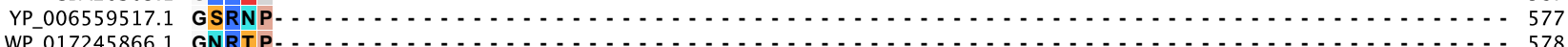

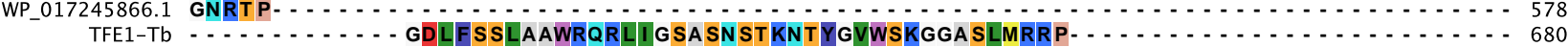

TFE1-TCO . . . . . . . . . . KGLLSVASAWQQRLTECFSCSGKNRAVAPVMRSSPLLHAP . . . . . . . . . . . . . . . . . . . . 679

TFE1-TCCLB _... . . . . . . GTFFNSIVTKLRGAKRAENEEGVAALGVWRKGGASLKRVP. . . . . . . . . . . . . . . . . . 670

TFE1-Lm QEASLEYRLRHPI A SWLWTGSAPNRLGTGSGDKGGSAAGTTT T AGCAAMLSDAATTPSVAASSAASRQRYPLVSLASL IR 810

TFE1-LmeX QEASLGYRLRHPIA SWLWT GSAPNRLGTGNSANRRSAAGATTAASCVPMLSDTATT SAVAASSAASRQRFPLVSLSSLIL 810

TFE1-Lt QEARLGYRLRHPI ESWVWT GSTRKHVDTGVSVKAGFSVGAANPAVCVPLVSNAATMSAVTASSTAPHQRYPLVSLASLVL 810

TFE1-Lb KDASLGYRLRHPI ASWLSTRSGTDNVGTGSAAKGGAAAGITNASDCALSLSDPSMT SAMAASSVASRHLYPLVSLT SLVL 810

Consensus $\mathbf{G K}$ -

Conservation

Sequence logo $0 \%$
$.3 \mathrm{bits}$

ABE53312.1 KKD IDATSYQLLGAAFGELKA - - FEADD I| IARCM I PM I I ET VRCLEEG I I ASPAEGDMGLVYGLGFPPFRGGVFRY IDTM 704 WP_017223439.1 QKT PEAKSYELLAEVAGEKAD- - FTKDD I I ARCM I PM INEVVRCLEEGIVATPGDADMALI YGIGFPPFRGGVFRYLDTM 678 WP_000965907.1 KKEEDAAVEDLLAEVSQPKRD- - FSEEEI I ARMM I PMVNEVVRCLEEGI I ATPAEADMALVYGLGFPPFHGGAFRWLDTL 678 WP_017005631.1 KKDVDAEVSPLLSTVVGKNTD- - YSDEEI I NRMMVPM INEVVRCLEEGI I ATPQEADMALVYGLGFPPFRGGVFRYLDT I 678

YP_433438.1 AKHLWPQLVEKYTDM- TKTQN- - TDLQELKDRLLFIQSLETMRCLEENVLRSVKDANIGSIFGLGFAPWT GGAIQYANQY 680 YP 003812264.1 KKHLWSGLKELFN - - KADAS- - I PFEDMGDRFLFIQSLEAVRCMEEGVI ERVADAN I GS IMGI GFPPWT GGV IQFI NGY 678 YP_003290744.1 PKYLWPELRRLFP. - - PQGEP. - LPQQEMIDRLLFVQALETVRCLDEGVLTSVADAN I GSVFGWGFAPFHGGTLQFI NAY 683 YP_001411745.1 KKKLWPGLADLAGKS-QQDPD- - LDVQELKNRFLY IQALEAARCFEEGVVTDVRDADVGA ILGWGFAPWAGGPLSLIDMV 701 TFE2-Lm - - AGLNSAVLQKYLGEELSAS- - I SETEIVDRCVLLMVNEATRI LDEGIAMSPEDVDTGMVWGTGFPPFRGGLLQYADHR 669

TFE2-Lmex - AGLNDGLLQKYLGKELSAS- - VSKTEIVDRCVLLMVNEATRI LDEGIAMSPEDVDTGMVWGTGFPPFRGGLLQYADHR 669 TFE2-Lb - MGLNNAVLKKYLGEELSPS- - I SETEIVDRCVLLMVNEATLI LDEA I AMSPEDVDTGMVWGTGFPAFRGGLLQYADHR 669 TFE2-LT - - AGLNNAALQKYI GGELSAS- - ISETEIVDRCVLLMVNEAARILDEGIVMSSEDVDTGMVWGTGFPPFRGGLLQYADHR 669

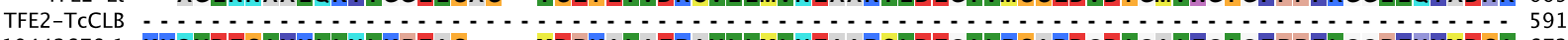

WP_019442678.1 KKQVDESIYKLLNLKPEAS - - - MDPKALAERAVLLMLNEAARCLDEGILRSARDGDIGAIFGIGFPPFLGGPFHYMDSI 672

NP_718651.1 - KAVDETVYGVLGIKPGVD - - - KEMSAVAERCVVQMLNEAVRCLDAGI I ASPRDGD I GAI FGIGFPPFLGGPFHYIDTL 670 YP_002416486.1 - KEVDKSVYKLLKLQPEPK- - - LSDND I TMRCVLPMLNEAVRCLDDGIIIRPRDGDIGA IFGI GFPPFLGGPFRYMDQ I 710 JW2338 KKQVDPA IYPLIGTQGQGR - - - ISAPQVAERCVMLMLNEAVRCVDEQVIRSVRDGDIGAVFGIGFPPFLGGPFRYIDSL 669

YP_633521.1 - QEVDSSI YALLPHGTERRS- - - FDRAEMAERVVLQMVNEA IRCLGEGILRSARDGDVGAIFGLGFPPFLGGPFHYVDSR 707 EFN74066.1-EK NREVNVGALDILK- KYTLEPKGST SDEDRQLRMVSRFVNEAVLCLQEN LANPLEGNVGAVFGLGFPPFTGGPFRWVDA 726 XP_001988242.1-EK SRPINTEALEIVKQKYSLDSKGANT PEDMTLRLVSRFVNEAVLCLEEKILDSPLEGDVGAVFGLGFPPFTGGPFRWVDQY 739

P40939-EK RKDLNSDMDSILASLKLPPKSEVSSDEDIQFRLVTRFVNEAVMCLQEGILATPAEGDI GAVFGLGFPPCLGGPFRFVDLY 724

BAB23628.1-EK KPDPWLSEFLSQYRETHHIKQRSI SKEEI LERCLYSLI NEAFRILEEGMAASPEHIDVI YLHGYGWPRHVGGPMYYAASV 665 XP_545234.1-EK KPDPWLSEFLSQYRKT YHI EPRI I SQDEI LERCLYSLI NEAFRI LGEGMATDPEH IDVVYLHGYGWPRHRGGPMFYASTV 670 NP_996951.1-EK KPDPLI QNLLETYRSRYGIQPRKI TDQEI I ERCLFALANEGFRILKDKI AGQPEDIDVI YLFGYGFPRHRGGPMFYA SMV 665

WP_008448388.1 YPNEQVNAM I VQHSADI GVARRK I SDQEI VERLVYSMVNEAAH I LEEGI ALRASD IDMVYLTGYGFPLFRGGPMFYADTV 656

YP_005996751.1 YPSQEVNDM I LQHSKDLGI TRRKI SDEEIVERLVFALVNEGAKILEEGI ASKASDI DMVYLTGYGFPLFRGGPMLYADTV 654

CBA26305.1 I PNAEVVAMI EEHRKALGITPRKI SDEEIVQRLVFSLVNEAAH I LEEGIAAKASDIDIVYI FGYGFPAHRGGPMNYADEV 667 YP_006559517.1 VPDPEVERI I EDFRKEQDI SSREI TDQEI LERCMYVMVNEAAKI LEEGI ADRALDIDVVWI YGYGFPAYRGGPMFWADQV 657 WP_017245866.1 QENPDLAPMLEAASREKGIERQALDEQYIVERCI FALVNEGAKILEEGIAQRSSD IDVIYLNGYGFPAFRGGPMYYADSV 658 TFE1-Tb YQDRDVELLI LDVCREKK I MRRDI SRKEM I ERILFAAVNEAAMLLREEAVSSSSA I DIATTFGHGFPAWRGGLCYYADKF 760 TFE1-TCO QRNRDVELLTLRI CSQKN I MRRD I SKREI I ERLLFSAVNEAGTLLRENVVSRSSA ID.................

TFE1-TCCLB RPDRAVELLI LDVCRKKDILRRD ISEREI VERI IFA I I NEAAKLLGEGAVLSSADVDVAMTFGYGFPAWKGGVCYYADKF 750 TFE1-Lm QHNYSTEYRT IDCSKRRRVTRRPFCEEEI FERIVFVMINEAAK IMADGIVT SSAD IDCT SVYGFGFPAWKGGLCYYADHV 890

TFE1-LmeX QHNYSVEYRTIDCSKRRRVTRRPFCEEEIFERIVFVMVNEAAKIMADGIVTNSAD IDCT SVYGFGFPAWKGGLCYYADHV 890

TFE1-Lt QHNYSSEYRT IDCSKRRR I TRRPFREEEIFERIVFAMINEAAKIMAEGII TNAADIDCT SVYGLGFPAWKGGLCYYADHV 890 TFE1-Lb QHNYSIEYRTIDCSKRRRVTRRPFHEEEIFERIVFAMVNEAAKIIMADGVIIASSADIDCT SVYAFGFPAWKGGLCYYADHV 890

Consensus KKDVDVEXXLLLYSKXEX I TR - - I SEEE I VERCVFAMVNEAARXLEEG I AASPADIDXGAVFGXGFPPFRGGPFRYADXV

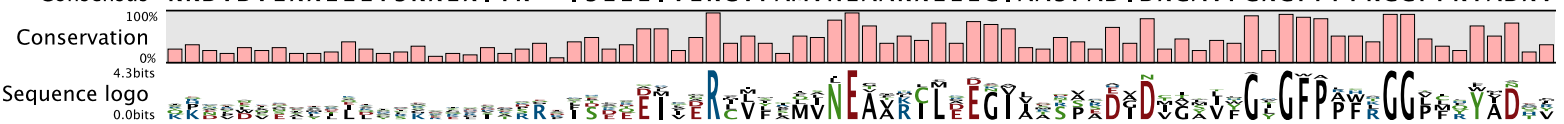




$$
980
$$

ABE53312.1 GVANFVALADKYAH. . LGGLYQVT- DAMRELAANNGSYYYQA . . . . . . . . . . . . 742

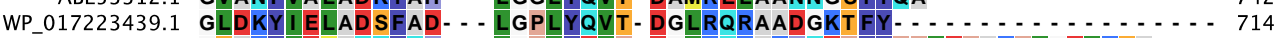
WP_000965907.1 GSAKYLDMAQQYQH - - - LGPLYEVP- EGLLRKARHNEPYYPPVEPARPVGDLKTA - . - 729 WP_017005631.1 GLANYVAMADKLAD - - LGPAYEIP- QGLRDKATQGEGYYDAQRQSA - . . . . . . . 721

YP 433438.1 GLRQFVERANYLRER - - HGERFTPP-ALLVSLAEKGAIFE- $\ldots \ldots \ldots \ldots$

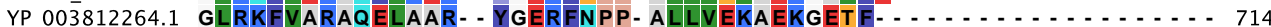

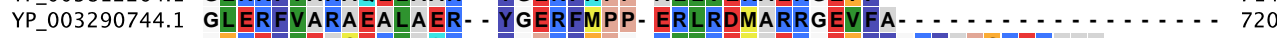
YP_001411745.1 GTKEFVEACDKLAQK - - YGPRFTPG- KLLRDMAEKGDTFYGRFAPSKEKAAA - . . . . . 750 TFE2-Lm GI ANVVAALEQLQRK- - TKRD YFAPTET LKKMA I EGKRFFPTRPYVPYQERHGYPKVTY 726

TFE2-Lmex GI ANVVAALEQLQRK- - TKKDYFAPT ET LKKMA I DGNRFFSTRPYVPYQERHGYPKVTY 726 TFE2-Lb GI ANVVAALEQLQSK- - TKKDYFAPTERLKKMAI KGMCFFPNRPYVPYQERHGYPKVTF 726 TFE2-Lt GIANVVAALEQLQRK- - TKKDHFAPTESLKKMAIEGKRFFPTRPYVPYQERHGYPKVTY 726

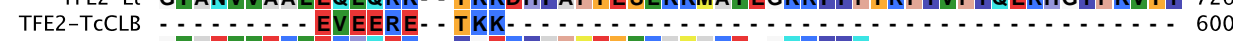
WP_019442678.1 GIAELVDKLERHQDK- - YGERFAPCESLKAMAKE- GQKFYN - . . . . . . . . . . . . . 710

NP 718651.1 GADNLVKILERYQTQ- - YGDRFEPCQRLKAMAAE- KARFF $\ldots \ldots \ldots$

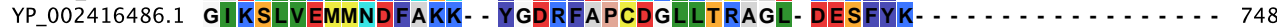

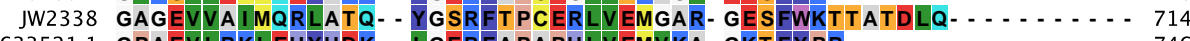

YP_633521.1 GPAEVLRKLEHYHDK- - LGERFAPAPHLVEMVKA- GKTFYPR - . . . . . . . . . . 746

EFN74066.1-EK GAHRFVKKMEEFQSH - YGESFRPCQLLLDMAADSTRKFHTK - . . . . . . . . . . . . 766 XP_001988242.1-EK GAGKLV SKMQSFAEL- - YGAPFKPSQTLLDMAKDPSKKFYPKT SDAKL- . . . . . . . . . - 785

P40939-EK GAQKIVDRLKKYEAA- - YGKQFTPCQLLADHANSPNKKFYQ - . . . . . . . . . . . 763 BAB23628.1-EK GLPTVLEKLQKYYRQ- NPDI PQLEPSDY LRRLVAQGSPPLKEWQSLAGPHSSKL- . . - . 718 XP_545234.1-EK GLPT VLEKLQKYYRQ- NPDI PQLEPCDYLKKLASLGNPPLKEWOSLAGPPGSKL- . - . - 723 NP_996951.1-EK GLERVLERLEYYHHA- LPDVPHLEPSPLLKKLVARGSPPIQKWREHIKSMHSHL- . . . . 718

WP_008448388.1 GLPNVLAAINKYAQG- - HQGGAWKPAPLLVRLAEEG- - . KGFNS - . - . . . . . . . . . . 695

YP_005996751.1 GLYNVAQAMRRYGKG- - YHGEAWKPAPLLQKLADAG - . . QGFNS - . . . . . . . . . . . . 693

CBA26305.1 GLFNVVQAMN RFAQNPLDDAKFWQPAPLLAKLAAEG $\ldots$ KTFN $\ldots \ldots \ldots \ldots \ldots$ YP_006559517.1 GLDVIILAAVEKYHAD - - VGGEQWKPADLLKQLVADG - . - KKFGDL - . . . . . . . . 697 WP_017245866.1 GLDKVLARVKELHAR- - CG- DWWKPAPLLEKLAAEG- . . - RTFTEWQAGQ- . . . . . . . 701

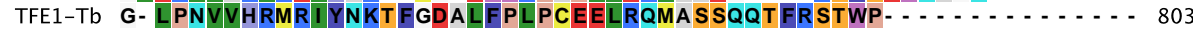

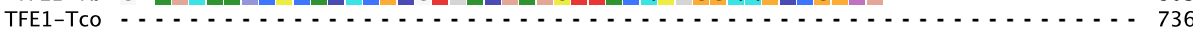

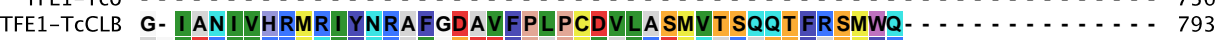

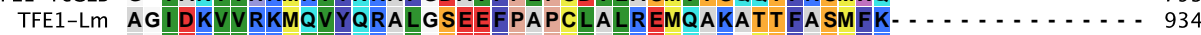

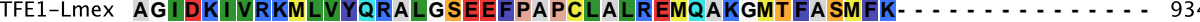

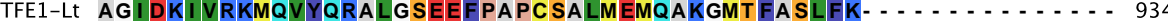
TFE1-Lb AGIDHIVQKMQVYQRALGSEEFPAPCLVLREMQAKGKTFATMFN $\ldots . . . . . . . . . .934$ Consensus GLANVVAXLEXXAQX - - YGERFPPP-ELLRKMAAKGKTFYK- - -

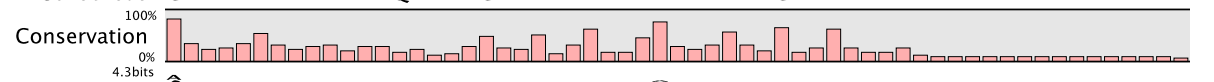

Sequence logo $\begin{aligned} & \text { l.3bits } \\ & \text { 0.0bits }\end{aligned}$ 